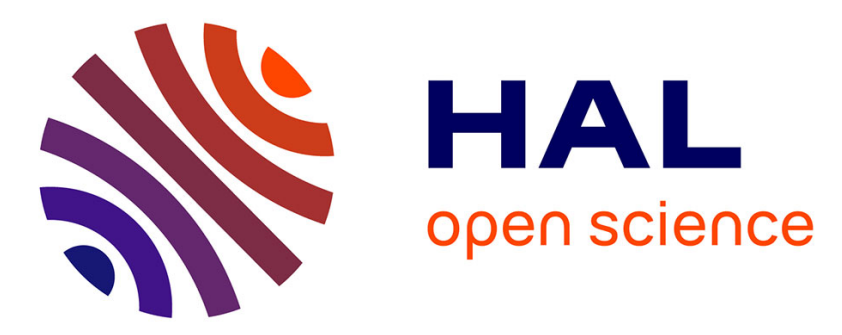

\title{
A new adaptive control chart for the simultaneous monitoring of the mean and variability of multivariate normal processes
}

\author{
Hamed Sabahno, Amirhossein Amiri, Philippe Castagliola
}

\section{To cite this version:}

Hamed Sabahno, Amirhossein Amiri, Philippe Castagliola. A new adaptive control chart for the simultaneous monitoring of the mean and variability of multivariate normal processes. Computers \& Industrial Engineering, 2021, 151, pp.106524. 10.1016/j.cie.2020.106524 · hal-03132610

\author{
HAL Id: hal-03132610 \\ https://hal.science/hal-03132610
}

Submitted on 12 Jul 2021

HAL is a multi-disciplinary open access archive for the deposit and dissemination of scientific research documents, whether they are published or not. The documents may come from teaching and research institutions in France or abroad, or from public or private research centers.
L'archive ouverte pluridisciplinaire HAL, est destinée au dépôt et à la diffusion de documents scientifiques de niveau recherche, publiés ou non, émanant des établissements d'enseignement et de recherche français ou étrangers, des laboratoires publics ou privés. 


\title{
A New Adaptive Control Chart for the Simultaneous Monitoring of the Mean and Variability of Multivariate Normal Processes
}

\author{
Hamed Sabahno ${ }^{\mathrm{a}}$, Amirhossein Amiri ${ }^{{ }^{*}}$ and Philippe Castagliola ${ }^{\mathrm{b}}$ \\ ${ }^{a}$ Department of Industrial Engineering, Faculty of Engineering, Shahed University, Tehran, Iran \\ ${ }^{b}$ Université de Nantes \& LS2N UMR CNRS 6004, Nantes, France \\ *Corresponding author: amiri@shahed.ac.ir
}

\begin{abstract}
This paper considers adaptive schemes for the simultaneous monitoring of the mean and variability of a multivariate normal quality characteristic. At first, we extend an already existing bivariate nonadaptive simultaneous control chart to a multivariate one. Then, we develop several adaptive schemes, which will cover both previously bivariate and newly multivariate charts. After having designed adaptive schemes for the multivariate chart, eight performance measures are computed based on the run length, time to signal, number of observations to signal and number of switches to signal and evaluated using a new Markov chain model. With the developed performance measures, non-adaptive and adaptive schemes under different mean, variability, simultaneous shift sizes, and different number of quality characteristics are compared. Our scheme is also compared to one of the best methods available in the literature. A numerical example is also provided in order to demonstrate how the adaptive scheme can be implemented in practice.
\end{abstract}

Keywords: Adaptive control charts, Simultaneous monitoring, Multivariate normal processes, Singlechart monitoring, Performance measures, Markov chains.

\section{Introduction}

Multivariate control charts are widely used in order to monitor various kinds of processes and to detect assignable causes, in the case of more than one quality characteristic. The multivariate Hotelling (1947)'s $T^{2}$ control chart is a very simple and effective tool to monitor the process mean vector when large shifts occur. However, in the case of small to moderate mean shifts, this Shewhart type control chart cannot perform properly. Allowing the chart to adapt based on the previous process states makes it capable to detect small and moderate process shifts more effectively. In adaptive control charts, at least one of the chart's parameters (sample size, sampling interval and probability of Type-I error) varies throughout the monitoring process and, based on that, adaptive charts can be divided into four main categories; VSS (Variable Sample Sizes), VSI (Variable Sampling Intervals), VSSI (Variable Sample Sizes and Sampling Intervals) and VP (Variable Parameters, if all of the chart parameters are allowed to vary). Researchers such as Aparisi (1996), Faraz and Moghadam (2009), Khoo et al. (2013) and Sabahno, Amiri and Castagliola (2019) and Chong et al. (2019) considered VSS type adaptive Hotelling's $T^{2}$ charts. For VSI type Hotelling's $T^{2}$ control charts we can refer to Aparisi and Haro (2001), Faraz et al. (2011) and Sabahno, Amiri and Castagliola (2018) for example. VSSI type Hotelling's $T^{2}$ control charts have been studied by authors such as Aparisi and Haro (2003) and Faraz and Parsian (2006). Finally, VP type ones have been investigated by authors such as Chen (2007), Faraz et al. (2014) and Seif et al. (2016). Researchers such as Du et al. (2012) proposed some approaches for classifying the source of the mean shift in the Hotelling's $T^{2}$ control chart. 
Multivariate control charts for monitoring the process variability have been considered by researchers as well. One of the common and simple method consists in using the sample's generalized variance $(|S|)$ defined as the determinant of the sample variance-covariance matrix. Alt (1985) proposed a bivariate control chart based on $|S|$. Aparisi et al. (1999) extended the work of Alt (1985) and they proposed a multivariate control chart either with upper and lower control limits or with an upper control limit only. Aparisi et al. (2001) developed a VSS scheme for the bivariate case of the generalized variance. They studied the performance of their control chart with the average run length criterion using a Markov chain approach. Grigoryan and He (2005) developed a multivariate double sampling (MDS) shewhart type $|S|$ control chart scheme in order to monitor the variability. Using a genetic algorithm combined with simulations, they showed that their new method performs better than the FP (Fixed Parameters) and VSS charts. Lee and Khoo. (2015) added a VSI feature to the multivariate synthetic shewart type $|S|$ control chart. Costa and Neto (2017) proposed the $S$ chart with variable charting statistic (called VCS $S$ chart) which is a Shewhart-type chart specially designed to control the covariance matrix of bi- and tri-variate processes. Though they found their method superior to the trivariate case, the only advantage of their method compared to the bi-variate generalized variance method was that it was easier to compute.

The simultaneous monitoring of the process parameters (mean and variability) instead of monitoring only one of them (which is more desired due to its simplicity), has caught some authors attention in the past years. The simultaneous monitoring has been proven to reduce the false alarm rates and to improve the performance of the monitoring procedure. In general, the simultaneous monitoring of control charts can be divided into single-chart and double-charts (one for each parameter) schemes. Concerning multivariate control charts for which two separate charts are used, one may refer to Yeh et al. (2003), Reynolds and Cho (2006), Hawkins and Maboudou-Tchao (2008) and Zhang and Chang (2008).

For multivariate single-chart schemes, the first contribution has been proposed by Yeh and Lin (2002). By using a probability integral transformation method, they introduced the box chart and they compared their single simultaneous chart with the traditional $T^{2}$ and $|S|$ (two) charts. However, they showed that their single chart, although simpler, does not usually perform better than the traditional $T^{2}$ and $|S|$ charts, especially in detecting small shifts. Khoo (2005) introduced a simple bivariate control chart for the simultaneous monitoring of the mean and variability. He used the Hotteling's $T^{2}$ statistic for the mean and Alt (1985)'s statistic, which is based on the generalized variance, for the variability. Then, based on those two statistics, he defined a new max-type statistic. Through simulations and by using the ARL performance measure, he evaluated the chart's performance under shifts in the mean, variability and both. Chen et al. (2005) also developed a single chart based on a max-type statistic containing two exponentially weighted moving average (EWMA) type statistics for each process parameters and they called it the Max-MEWMA chart. They also compared the performance of their chart to the traditional $T^{2}$ and $|S|$ charts and they concluded that the MaxMEWMA chart has better performances in detecting small to moderate shifts. Zhang et al. (2010) proposed a new single control chart which integrates an EWMA procedure with the generalized likelihood ratio (GLR) test and they called it ELR for jointly monitoring both multivariate process mean and variability. Their ELR chart, unlike the box and Max-MEWMA charts, can be applied for 
individual observations as well. Wang et al. (2014) developed two simultaneous monitoring charts i) based on the generalized likelihood ratio test (GLRT) called PGLRT (penalized GLRT) and ii) based on the multivariate exponentially weighted moving average and covariance (MEWMAC) called PMEWMAC (penalized MEWMAC). The PGLRT chart only uses a statistic while the other one uses two statistics. Both methods are only used for individual observations.

For adaptive simultaneous double-chart schemes, Reynolds and Kim (2007) investigated the option of using sequential sampling, with the sample size at each sampling point depending on the process data, for the Shewhart and MEWMA-types chart combinations. They showed that the sequential sampling provides significant improvements to the charts' performance under most process shifts. Reynolds and Cho (2011) extended the work of Reynolds and Kim (2007) by adding a VSI feature. To the best of the authors' knowledge, adaptive schemes for multivariate simultaneous single-chart schemes have not been considered yet. In order to summarize the literature review and to show the limitations of the papers, we have classified them in Table 1, in terms of the control chart type, the number of quality characteristics, whether they have adaptive features or not, whether they can simultaneously monitor the process mean and variability or not, and finally, the computation method of performance measure(s).

\section{"Insert Table 1 here"}

According to our literature review (Table 1), adaptive features in multivariate simultaneous charts have only been considered in two papers and they both used double-charts and also they both used simulation runs in order to measure the chart performance. Using simulation methods to measure the charts performance is often very time consuming. If the distributions of the statistics used for monitoring are available, then the Markov chain is always the preferable method for computing performance measures.

As mentioned above, the work of Khoo (2005), although very simple and innovative, presented a simultaneous scheme capable of monitoring processes with only two quality characteristics, which is a very limited situation and, generally speaking, products might have more than two quality characteristics to be monitored. In addition, it is assumed that all the chart parameters are fixed throughout the monitoring process and no adaptive strategies were used. He also only used the ARL as the chart performance measure, obtained through very time consuming simulation runs. In addition, his chart was very slow specially in detecting small shifts.

In this paper, we improve the innovative approach of Khoo (2005) in four directions: 1) We extend his bivariate-only method to a multivariate one in order to be able to monitor products with more than two quality characteristics. 2) We add adaptive monitoring strategies to both bivariate and multivariate charts to increase the charts performance in detecting shifts. 3) We use 7 extra performance measures (other than just the classical ARL previously used by him) to measure the charts performance in many aspects. 4) We use a new Markov chain model to compute the performance measures instantaneously and more accurately rather than the very time consuming simulation method used by him.

The structure of this paper is as follows. In Section 2, we develop a single-type chart for simultaneously monitoring the mean and variability of a multivariate normal characteristic. We add adaptive features to the chart in Section 3. The performance measures are developed using a Markov chains methodology in Section 4. In Section 5, we perform simulation studies in order to compare the charts' performances and also we present an industrial application. Concluding remarks and suggestions for future researches are discussed in Section 6. 


\section{Proposed Multivariate Control Charts for Simultaneous Monitoring of Mean and Variability}

When the in-control process parameters $\left(\boldsymbol{\mu}_{0}\right.$ and $\boldsymbol{\Sigma}_{0}$ ) are known, in order to monitor the process mean the Hotelling's $T^{2}$ statistic is evaluated for each subgroup $i=1,2,3, \ldots$ as:

$$
T_{i}^{2}=n\left(\overline{\mathbf{X}}_{i}-\boldsymbol{\mu}_{0}\right)^{T}\left(\Sigma_{0}\right)^{-1}\left(\overline{\mathbf{X}}_{i}-\boldsymbol{\mu}_{0}\right)
$$

where $n$ is the sample (subgroup) size, $\overline{\mathbf{X}}_{i}$ is the sample's mean and $T_{i}^{2} \sim \chi^{2}(p)$, i.e. a chi-square distribution with $p$ degrees of freedom and $p$ is the number of quality characteristics.

For the process variability, as suggested by Gnanadesikan and Gupta (1970), we use the following statistic:

$$
W_{i}=\frac{(n-1)\left|\mathbf{S}_{i}\right|^{1 / p}}{\left|\Sigma_{0}\right|^{1 / p}},
$$

which follows approximately a gamma distribution $\Gamma(a, b)$ for $p>2$, where $a=p(n-p) / 2$ and $b=\left(\frac{2}{P}\left(1-\frac{(p-1)(p-2)}{2 n}\right)^{-1 / p}\right.$. Note that $W_{i}$ exactly follows a gamma distribution for $p=1$ and $p=2$.

In order to monitor the mean and variability simultaneously, we use the following transformations of $T_{i}^{2}$ and $W_{i}$ :

$$
M_{i}=\Phi^{-1}\left[H_{p}\left(T_{i}^{2}\right)\right] \sim N(0,1), \quad i=1,2, \ldots
$$

and

$$
V_{i}=\Phi^{-1}\left[G_{(a, b)}\left(W_{i}\right)\right] \sim N(0,1), \quad i=1,2, \ldots,
$$

where $\Phi($.$) is the standard normal cumulative distribution function, H_{p}($.$) represents the chi-square$ cumulative distribution function with $p$ degrees of freedom and $G_{a, b}$ (.) denotes the gamma cumulative distribution function with shape parameter $a$ and scale parameter $b$.

Note that, Khoo (2005) used a similar approach to monitor bivariate quality characteristics. However, he used the following statistic $V_{i}=\Phi^{-1}\left[H_{2 n-4}\left(2 W_{i}\right)\right] \sim N(0,1), i=1,2, \ldots$, instead of the proposed statistic for monitoring the variability, where $2 W_{i}$ follows a chi-square distribution with $2 n-4$ degrees of freedom. Note that $p=2$ in the $2 W_{i}$ statistic by Khoo (2005).

Then, we use the following max-type statistic:

$$
C_{i}=\max \left\{\left|M_{i}\right|,\left|V_{i}\right|\right\} .
$$

The upper control limit (UCL) of this chart can be obtained by solving the following equation:

$$
\begin{aligned}
P\left(C_{i}\right. & \leq U C L)=P\left(\max \left\{\left|M_{i}\right|,\left|V_{i}\right|\right\} \leq U C L\right) \\
& =P\left(-U C L \leq M_{i} \leq U C L\right) P\left(-U C L \leq V_{i} \leq U C L\right) \\
& =(2 \Phi(U C L)-1)(2 \Phi(U C L)-1)=(2 \Phi(U C L)-1)^{2}=1-\alpha,
\end{aligned}
$$

where $\alpha$ is the probability of Type-I error. 


\section{Proposed Adaptive Multivariate Control Charts for Monitoring the Mean and Variability Simultaneously}

For adaptive control charts, at least one of the process parameters (sample size, sampling interval and probability of Type-I error) is allowed to vary throughout the sampling process. If all parameters vary, the adaptive chart is called a VP control chart. In this paper, we assume that there are:

- two types of sample sizes $\left(n_{s}: s \in\{1,2\}\right.$ with $\left.n_{1}<n_{2}\right)$,

- two types of sampling intervals $\left(t_{s}: s \in\{1,2\}\right.$ with $\left.t_{2}<t_{1}\right)$,

- two types of type I error probabilities $\left(\alpha_{s}: s \in\{1,2\}\right.$ where $\left.\alpha_{1}<\alpha_{2}\right)$,

and, as our max-type control chart is one-sided, we also assume that there are:

- two upper control limits $U C L_{1}\left(\right.$ related to $\alpha_{1}$ ) and $U C L_{2}\left(\right.$ related to $\alpha_{2}$ ) As $\alpha_{1}<\alpha_{2}$ we have $U C L_{2}<U C L_{1}$.

- two upper warning limits $U W L_{1}$ and $U W L_{2}$ verifying $U W L_{1}<U C L_{1}$ and $U W L_{2}<U C L_{2}$, respectively.

The VP strategy for choosing the next sampling scheme is as follows:

- If $C_{i} \in[0, U W L]$, the process is declared as being in-control and the parameters for the next sample must be $\Omega_{1}=\left(n_{1}, t_{1}, U C L_{1}, U W L_{1}\right)$, i.e. the small sample size, the long sampling interval and the large control and warning limits $U C L$ and $U W L$.

- If $C_{i} \in(U W L, U C L]$, the process is also declared as being in-control but the parameters for the next sample must be $\Omega_{2}=\left(n_{2}, t_{2}, U C L_{2}, U W L_{2}\right)$, i.e. the large sample size, the short sampling interval and the small control and warning limits $U C L$ and $U W L$.

- If $C_{i} \in(U C L, \infty)$, the process is declared as being out-of-control and corrective actions have to be taken.

In the VP scheme, the following equations should be satisfied.

$$
\begin{gathered}
A S S=n_{1} P_{0}+n_{2}\left(1-P_{0}\right), \\
A S I=t_{1} P_{0}+t_{2}\left(1-P_{0}\right), \\
A T E=\alpha_{1} P_{0}+\alpha_{2}\left(1-P_{0}\right),
\end{gathered}
$$

where $A S S$ is the average sample size, $A S I$ is the average sampling interval and ATE is the average Type-I error where $P_{0}$ is the probability of being in the safe state $\left(\Omega_{1}\right)$ while the process is in-control, i.e.:

$$
\begin{aligned}
P_{0} & =\frac{P\left(C_{i} \leq U W L\right)}{P\left(C_{i} \leq U C L\right)}=\frac{P\left(\max \left\{\left|M_{i}\right|,\left|V_{i}\right|\right\} \leq U W L\right)}{P\left(\max \left\{\left|M_{i}\right|,\left|V_{i}\right|\right\} \leq U C L\right)} \\
& =\frac{P\left(\left|M_{i}\right| \leq U W L\right) P\left(V_{i} \mid \leq U W L\right)}{P\left(\left|M_{i}\right| \leq U C L\right) P\left(\left|V_{i}\right| \leq U C L\right)}=\frac{[2 \Phi(U W L)-1]^{2}}{[2 \Phi(U C L)-1]^{2}}
\end{aligned}
$$

Note that for a VSS scheme, only Equation (7) must be kept and Equations (8) and (9) must be disregarded. Similarly, for a VSI scheme, only Equation (8) must be kept and Equations (7) and (9) have to be disregarded. Finally, for a VSSI scheme, only Equations (7) and (8) must be kept and Equation (9) must be ignored. 
In order to solve Equations (7) to (9), some of the parameters' values should be fixed and some others have to be computed. The following steps can be taken to obtain the parameters' values.

Step 1. Fix $\alpha_{1}$ and, using Equation (6), $U C L_{1}$ is obtained as:

$$
U C L_{1}=\Phi^{-1}\left(\frac{\sqrt{1-\alpha_{1}}+1}{2}\right)
$$

Step 2. Fix $A S S, n_{1}, n_{2}$ and, using Equation (7), $P_{0}$ is obtained as:

$$
P_{0}=\frac{A S S-n_{2}}{n_{1}-n_{2}} .
$$

Step 3. Fix ATE and, using Equations (9) and (12), $\alpha_{2}$ is obtained as:

$$
\alpha_{2}=\frac{A T E\left(n_{1}-n_{2}\right)-\alpha_{1}\left(A S S-n_{2}\right)}{n_{1}-A S S}
$$

and $U C L_{2}$ is deduced from Equation (6) as:

$$
U C L_{2}=\Phi^{-1}\left(\frac{\sqrt{1-\alpha_{2}}+1}{2}\right) .
$$

Step 4. Fix $A S I, t_{2}$ and, using Equations (8) and (12), $t_{1}$ is obtained as:

$$
t_{1}=\frac{A S I\left(n_{1}-n_{2}\right)-t_{2}\left(n_{1}-A S S\right)}{A S S-n_{2}} .
$$

Step 5. By using Equation (10), $U W L_{1}$ and $U W L_{2}$ are obtained as follows:

$$
U W L_{s}=\Phi^{-1}\left(\frac{\left(2 \Phi\left(U C L_{s}\right)-1\right) \sqrt{P_{0}}+1}{2}\right)
$$

\section{Performance measures}

For a FP control chart (for which the chart parameters are all fixed), performance measures like the Average Run Length (ARL) and the Standard Deviation of Run Length (SDRL) can be used and investigated. However, for adaptive schemes, six additional performance measures can be used: the Average Time to Signal (ATS), the Standard Deviation of Time to Signal (SDTS), the Average Number of Observations to Signal (ANOS), the Standard Deviation of Number of Observations to Signal (SDNOS), the Average Number of Switches to Signal (ANSW) and the Standard Deviation of Number of Switches to Signal (SDNSW).

The ARL and SDRL are the average and standard deviation of the number of samples taken before an out-of-control signal occurs, the ATS and SDTS are the average and standard deviation of the time needed until a control chart signals, the ANOS and SDNOS are the average and standard deviation of the number of products observed before an out-of-control signal and, finally, the ANSW and SDNSW are the average and standard deviation of the number of switches from $\Omega_{1} / \Omega_{2}$ to $\Omega_{2} / \Omega_{1}$ while the process is in-control. If the number of switches increases, the difficulty of the chart's administration increases as well. This is why these performance measures are worth to be investigated. Since there is 
no switching in FP control charts, they always have an advantage in this regard and can be easily administrated.

It is reasonable to say that the most important criteria in order to measure the charts' performance is the time to signal (ATS/SDTS) and all charts' performances should be compared primarily via this time to signal criteria.

When the process is out-of-control, smaller ARL, ATS and ANOS values are preferable. On the contrary, when the process is in-control, larger ARL, ATS and ANOS values are expected. However, no matter if the process is in- or out-of-control, smaller SDRL, SDTS, SDNOS, ANSW and SDNSW values are always expected, especially in out-of-control situations.

In order to compute these quantities, we use a Markov Chain model. This approach requires to define the following three states:

$$
\text { State } 1: C_{i} \in[0, U W L]
$$

State 2: $C_{i} \in(U W L, U C L]$,

$$
\text { State 3: } C_{i} \in(U C L, \infty) \text {. }
$$

The first two states are transient while, the third one is absorbing. The Markov transition probability matrix $\mathbf{P}$ corresponding to the VP chart is equal to

$$
\mathbf{P}=\left[\begin{array}{lll}
p_{11} & p_{12} & p_{13} \\
p_{21} & p_{22} & p_{23} \\
p_{31} & p_{32} & p_{33}
\end{array}\right],
$$

where $p_{i j}$ is the probability of transition from state $i$ to state $j$.

Since by definition $T_{i}^{2}$ and $W_{i}$ are independent for a multivariate normal characteristic, consequently, $M_{i}$ and $V_{i}$ are also independent. Therefore, the transient state probabilities $p_{11}, p_{12}, p_{21}$ and $p_{22}$ for the proposed multivariate max-type chart are equal to:

$$
\begin{aligned}
p_{s 1}= & P\left(C_{i} \leq U W L\right)=P\left(\max \left\{\left|M_{i}\right|,\left|V_{i}\right|\right\} \leq U W L\right) \\
= & P\left(-U W L \leq M_{i} \leq U W L\right) P\left(-U W L \leq V_{i} \leq U W L\right), \\
p_{s 2}= & P\left(U W L<C_{i} \leq U C L\right)=P\left(U W L<\max \left\{\left|M_{i}\right|,\left|V_{i}\right|\right\} \leq U C L\right) \\
= & P\left(-U C L \leq M_{i}<-U W L\right) P\left(-U C L \leq V_{i}<-U W L\right)+ \\
& P\left(-U C L \leq M_{i}<-U W L\right) P\left(-U W L<V_{i}<U W L\right)+ \\
& P\left(-U C L \leq M_{i}<-U W L\right) P\left(U W L<V_{i} \leq U C L\right)+ \\
& P\left(-U W L<M_{i}<U W L\right) P\left(-U C L \leq V_{i}<-U W L\right)+ \\
& P\left(-U W L<M_{i}<U W L\right) P\left(U W L<V_{i} \leq U C L\right)+ \\
& P\left(U W L<M_{i} \leq U C L\right) P\left(U W L<V_{i} \leq U C L\right)+ \\
& P\left(U W L<M_{i} \leq U C L\right) P\left(-U W L<V_{i}<U W L\right)+ \\
& P\left(U W L<M_{i} \leq U C L\right) P\left(-U C L \leq V_{i}<-U W L\right) .
\end{aligned}
$$

Concerning the other probabilities of transition, we simply have $p_{13}=1-p_{11}-p_{12}, p_{23}=1-p_{21}-p_{22}$, $p_{31}=p_{32}=0$ and $p_{33}=1$. 
As it can be seen, Equations (17) and (18) are both sums of products $P\left(m_{1} \leq M_{i} \leq m_{2}\right)$ by $P\left(v_{1} \leq V_{i} \leq v_{2}\right)$ where $m_{1}, m_{2}, v_{1}$ and $v_{2}$ stand for +/-UWL and +/-UCL. In general, these terms can be evaluated using Equations (21) and (23) when the process is out-of-control and using Equations (22) and (24) when the process is in-control. Let us first derive Equations (21) and (22) for $P\left(m_{1} \leq M_{i} \leq m_{2}\right)$. We have:

$$
\begin{aligned}
P\left(m_{1}\right. & \left.\leq M_{i} \leq m_{2}\right)=P\left(m_{1} \leq \Phi^{-1}\left[H_{p}\left(T_{i}^{2}\right)\right] \leq m_{2}\right) \\
& =P\left(\Phi\left(m_{1}\right) \leq\left[H_{p}\left(T_{i}^{2}\right)\right] \leq \Phi\left(m_{2}\right)\right) \\
& =P\left(H_{p}^{-1}\left(\Phi\left(m_{1}\right)\right) \leq T_{i}^{2} \leq H_{p}^{-1}\left(\Phi\left(m_{2}\right)\right)\right) .
\end{aligned}
$$

In order to compute Equation (19), let us assume that $p>1$, i.e. $\mathrm{X}_{j} \sim \operatorname{MN}(\boldsymbol{\mu}, \boldsymbol{\Sigma}), j=1,2, \ldots, n_{s}$. When the process is in-control, we have $\mu=\mu_{0}$ and $\Sigma=\Sigma_{0}$. Let

$$
Z_{i}=\sqrt{n_{s}} \Sigma_{0}^{-1 / 2}\left(\overline{\mathbf{X}}_{i}-\mu_{0}\right)
$$

where $\Sigma_{0}{ }^{-1 / 2}$ is the matrix square root of the inverse of $\Sigma_{0}$, i.e. $\Sigma_{0}{ }^{-1 / 2} \Sigma_{0}{ }^{-1 / 2}=\Sigma_{0}{ }^{-1}$. When the process is in-control, we have $\mathbf{Z}_{i} \sim \operatorname{MN}(\mathbf{0}, \mathbf{I})$ and, therefore $T_{i}^{2}=\mathbf{Z}_{i}^{T} \mathbf{Z}_{i} \sim \chi^{2}(p)$.

When the process is out-of-control, we have $\mu=\mu_{1}, \Sigma=\Sigma_{1}$ and the statistic

$$
\mathbf{Z}_{i} \sim \mathrm{MN}\left(\sqrt{n_{s}} \Sigma_{0}^{-1 / 2}\left(\mu_{1}-\mu_{0}\right), \Sigma_{0}^{-1 / 2} \Sigma_{1} \Sigma_{0}^{-1 / 2}\right) .
$$

Let us define $\boldsymbol{\Lambda}=\Sigma_{0}{ }^{-1 / 2} \Sigma_{1} \Sigma_{0}{ }^{-1 / 2}$ and we deduce

Consequently, we have

$$
\Lambda^{-1 / 2}=\left(\left(\Sigma_{0}^{-1 / 2} \Sigma_{1} \Sigma_{0}^{-1 / 2}\right)^{-1}\right)^{1 / 2}=\left(\Sigma_{0}^{1 / 2} \Sigma_{1}^{-1} \Sigma_{0}^{1 / 2}\right)^{1 / 2} .
$$

and

$$
\boldsymbol{\Lambda}^{-1 / 2} \mathbf{Z}_{i} \sim \mathrm{MN}\left(\sqrt{n_{s}} \boldsymbol{\Lambda}^{-1 / 2} \boldsymbol{\Sigma}_{0}^{-1 / 2}\left(\boldsymbol{\mu}_{1}-\boldsymbol{\mu}_{0}\right), \mathbf{I}\right)
$$

$$
\mathbf{Z}_{i}^{T} \boldsymbol{\Lambda}^{-1 / 2} \boldsymbol{\Lambda}^{-1 / 2} \mathbf{Z}_{i}=\mathbf{Z}_{i}^{T} \boldsymbol{\Lambda}^{-1} \mathbf{Z}_{i} \sim \chi^{2}(p, \lambda),
$$

With the noncentrality parameter $\lambda$ equals to

$$
\begin{aligned}
\lambda & =n_{s}\left(\boldsymbol{\mu}_{1}-\boldsymbol{\mu}_{0}\right)^{T} \boldsymbol{\Sigma}_{0}{ }^{-1 / 2} \boldsymbol{\Lambda}^{-1 / 2} \boldsymbol{\Lambda}^{-1 / 2} \boldsymbol{\Sigma}_{0}^{-1 / 2}\left(\boldsymbol{\mu}_{1}-\boldsymbol{\mu}_{0}\right) \\
& =n_{s}\left(\boldsymbol{\mu}_{1}-\boldsymbol{\mu}_{0}\right)^{T} \boldsymbol{\Sigma}_{0}{ }^{-1 / 2} \boldsymbol{\Lambda}^{-1} \boldsymbol{\Sigma}_{0}^{-1 / 2}\left(\boldsymbol{\mu}_{1}-\boldsymbol{\mu}_{0}\right) .
\end{aligned}
$$

If we replace $\boldsymbol{\Lambda}$ with $\Sigma_{0}{ }^{-1 / 2} \Sigma_{1} \Sigma_{0}{ }^{-1 / 2}$, we have $\boldsymbol{\Lambda}^{-1}=\Sigma_{0}{ }^{1 / 2} \Sigma_{1}{ }^{-1} \Sigma_{0}{ }^{1 / 2}$ and the noncentrality parameter simplifies to

$$
\lambda=n_{s}\left(\boldsymbol{\mu}_{1}-\boldsymbol{\mu}_{0}\right)^{T}\left(\boldsymbol{\Sigma}_{1}\right)^{-1}\left(\boldsymbol{\mu}_{1}-\boldsymbol{\mu}_{0}\right) .
$$

As it is mentioned above, for general variance-covariance matrices $\boldsymbol{\Sigma}_{0}$ and $\boldsymbol{\Sigma}_{1}$, we have $\mathbf{Z}_{i}^{T} \boldsymbol{\Lambda}^{-1} \mathbf{Z}_{i} \sim \chi^{2}(p, \lambda)$. However, in order to evaluate the terms $P\left(m_{1} \leq M_{i} \leq m_{2}\right)$ in Equation (19), it is impossible to obtain the distribution of $T_{i}^{2}=\mathbf{Z}_{i}^{T} \mathbf{Z}_{i}$ for an out-of-control condition. To overcome this problem, we will assume that $\boldsymbol{\Sigma}_{1}=\tau \boldsymbol{\Sigma}_{0}$, with $\tau>0$. Therefore, we have $\boldsymbol{\Lambda}^{-1}=\frac{1}{\tau} \mathbf{I}$ where $\mathbf{I}$ is the identity matrix and, consequently, $\frac{1}{\tau} T_{i}^{2}=\frac{1}{\tau} \mathbf{Z}_{i}^{T} \mathbf{Z}_{i} \sim \chi^{2}(p, \lambda)$ with $\lambda=\frac{n_{s}}{\tau}\left(\boldsymbol{\mu}_{1}-\boldsymbol{\mu}_{0}\right)^{T}\left(\boldsymbol{\Sigma}_{0}\right)^{-1}\left(\boldsymbol{\mu}_{1}-\boldsymbol{\mu}_{0}\right)$.

As a result, we have

$$
P\left(m_{1} \leq M_{i} \leq m_{2}\right)=H_{p, \lambda}\left(H_{p}^{-1}\left(\Phi\left(m_{2}\right)\right) \times(1 / \tau)\right)-H_{p, \lambda}\left(H_{p}^{-1}\left(\Phi\left(m_{1}\right)\right) \times(1 / \tau)\right) .
$$

When the process is in-control, i.e. $\lambda=0$ and $\boldsymbol{\Sigma}_{1}=\boldsymbol{\Sigma}_{0}$, the previous equation reduces to: 


$$
P\left(m_{1} \leq M_{i} \leq m_{2}\right)=H_{p}\left(H_{p}^{-1}\left(\Phi\left(m_{2}\right)\right)\right)-H_{p}\left(H_{p}^{-1}\left(\Phi\left(m_{1}\right)\right)\right)=\Phi\left(m_{2}\right)-\Phi\left(m_{1}\right)
$$

Similarly, for $P\left(v_{1} \leq V_{i} \leq v_{2}\right)$, we have:

$$
\begin{aligned}
P\left(v_{1}\right. & \left.\leq V_{i} \leq v_{2}\right)=P\left(v_{1} \leq \Phi^{-1}\left[G_{(a, b)}\left(W_{i}\right)\right] \leq v_{2}\right) \\
& =P\left(\Phi\left(\left(v_{1}\right) \leq\left[G_{(a, b)}\left(W_{i}\right)\right] \leq \Phi\left(v_{2}\right)\right)\right. \\
& =P\left(G_{(a, b)}^{-1}\left(\Phi\left(v_{1}\right)\right) \leq W_{i} \leq G_{(a, b)}^{-1}\left(\Phi\left(v_{2}\right)\right)\right)
\end{aligned}
$$

In order to obtain the distribution of $W_{i}$ when the process is out-of-control we multiply in the previous equation $\left|\boldsymbol{\Sigma}_{0}\right|$ by $\left|\boldsymbol{\Sigma}_{1}\right| /\left|\boldsymbol{\Sigma}_{1}\right|=1$, i.e.

$$
P\left(G_{(a, b)}^{-1}\left(\Phi\left(v_{1}\right)\right) \leq \frac{\left(n_{s}-1\right)\left|S_{i}\right|^{1 / p}}{\left[\left|\boldsymbol{\Sigma}_{0}\right|\left|\boldsymbol{\Sigma}_{1}\right| /\left|\boldsymbol{\Sigma}_{1}\right|\right]^{1 / p}} \leq G_{(a, b)}^{-1}\left(\Phi\left(v_{2}\right)\right)\right) .
$$

Finally, all the terms are multiplied by $\left[\left|\boldsymbol{\Sigma}_{0}\right| /\left|\boldsymbol{\Sigma}_{1}\right|\right]^{1 / p}$ and as a result, we have

$$
\begin{aligned}
& P\left(v_{1} \leq V_{i} \leq v_{2}\right)= \\
& G_{(a, b)}\left(G_{(a, b)}^{-1}\left(\Phi\left(v_{2}\right)\right) \times\left[\left|\boldsymbol{\Sigma}_{0}\right| /\left|\boldsymbol{\Sigma}_{1}\right|\right]^{1 / p}\right)-G_{(a, b)}\left(G_{(a, b)}^{-1}\left(\Phi\left(v_{1}\right)\right) \times\left[\left|\boldsymbol{\Sigma}_{0}\right| /\left|\boldsymbol{\Sigma}_{1}\right|\right]^{1 / p}\right) .
\end{aligned}
$$

When the process is in-control, i.e. $\boldsymbol{\Sigma}_{1}=\boldsymbol{\Sigma}_{0}$, the previous equation reduces to:

$$
\begin{aligned}
P\left(v_{1} \leq V_{i} \leq v_{2}\right)= & G_{(a, b)}\left(G_{(a, b)}^{-1}\left(\Phi\left(v_{2}\right)\right)\right)-G_{(a, b)}\left(G_{(a, b)}^{-1}\left(\Phi\left(v_{1}\right)\right)\right) \\
& =\Phi\left(v_{2}\right)-\Phi\left(v_{1}\right) .
\end{aligned}
$$

One can easily compute Equations (17) and (18) using terms $P\left(m_{1} \leq M_{i} \leq m_{2}\right)$ and $P\left(v_{1} \leq V_{i} \leq v_{2}\right)$, replacing $m_{1}, m_{2}, v_{1}$ and $v_{2}$ in Equations (21) - (24) with $\pm U W L$ and $\pm U C L$.

In this paper, we consider two scenarios for the shift in the variance-covariance matrix as follows:

- Scenario 1- In this scenario we assume that all the elements (variance and covariance values) in $\boldsymbol{\Sigma}_{0}$ shift with an equal multiplier $\tau$.

- Scenario 2- In this scenario, each element of the variance-covariance matrix shifts independently and not necessarily in an equal way. In this case, we again consider the general form $\boldsymbol{\Sigma}_{1}=\tau \boldsymbol{\Sigma}_{0}$ and estimate $\tau$ as $|\Lambda|^{1 / p}$ through simulation runs. Note that $\tau$ is estimated such that the relationship $\boldsymbol{\Sigma}_{1}=\tau \boldsymbol{\Sigma}_{0}$ is nearly satisfied. In this scenario, $\boldsymbol{\Sigma}_{0}$ and $\boldsymbol{\Sigma}_{1}$ can be any matrices.

Note that, the scenario 1 is a special case of scenario 2 .

Then, from Jensen et al. (2008), we have the general forms of the performance measures as:

$$
\begin{gathered}
\mathrm{ARL}=\mathbf{b}^{\mathrm{T}}(\mathbf{I}-\mathbf{Q})^{-1} \mathbf{1}, \\
\mathrm{SDRL}=\sqrt{\mathbf{b}^{\mathrm{T}}(\mathbf{I}-\mathbf{Q})^{-1}\left(2 \mathbf{I}(\mathbf{I}-\mathbf{Q})^{-1} \mathbf{1}-\mathbf{1}\right)-(\mathrm{ARL})^{2}}, \\
\mathrm{ATS}=\mathbf{b}^{\mathrm{T}}(\mathbf{I}-\mathbf{Q})^{-1} \mathbf{t},
\end{gathered}
$$




$$
\begin{gathered}
\operatorname{SDTS}=\sqrt{\mathbf{b}^{\mathrm{T}}(\mathbf{I}-\mathbf{Q})^{-1}\left(2 \mathbf{D}_{\mathbf{t}}(\mathbf{I}-\mathbf{Q})^{-1} \mathbf{t}-\mathbf{t}^{(2)}\right)-(\mathrm{ATS})^{2}}, \\
\operatorname{ANOS}=\mathbf{b}^{\mathrm{T}}(\mathbf{I}-\mathbf{Q})^{-1} \mathbf{n}, \\
\operatorname{ANNOS}=\sqrt{\mathbf{b}^{\mathrm{T}}(\mathbf{I}-\mathbf{Q})^{-1}\left(2 \mathbf{D}_{\mathbf{n}}(\mathbf{I}-\mathbf{Q})^{-1} \mathbf{n}-\mathbf{n}^{(2)}\right)-(\mathrm{ANOS})^{2},} \\
\operatorname{SDNSW}=\sqrt{\mathbf{b}^{\mathrm{T}}(\mathbf{I}-\mathbf{Q})^{-1}\left(2 \mathbf{D}_{\mathbf{p}}(\mathbf{I}-\mathbf{Q})^{-1} \mathbf{p}-\mathbf{p}^{(2)}\right)-(\mathrm{I}-\mathbf{Q})^{-1} \mathbf{p},}
\end{gathered}
$$

where $\mathbf{b}^{\mathrm{T}}=\left(b_{1}, b_{2}\right)$ is the vector of starting probabilities such that $b_{1}+b_{2}=1$, $\mathbf{I}$ is the identity matrix of order $2, \mathbf{Q}$ is a $2 \times 2$ transition probability matrix for the transient states, $\mathbf{1}$ is a $2 \times 1$ unit column vector, $\mathbf{t}=\left(t_{1}, t_{2}\right)^{\mathrm{T}}$ is the vector of sample sizes, $\mathbf{D}_{\mathbf{t}}$ is the $2 \times 2$ diagonal matrix with the diagonal elements of $\mathbf{t}, \mathbf{t}^{(2)}$ contains the squares of the elements of vector $\mathbf{t}, \mathbf{n}=\left(n_{1}, n_{2}\right)^{\mathrm{T}}$ is the vector of sample sizes, $\mathbf{D}_{\mathbf{n}}$ is the $2 \times 2$ diagonal matrix with the diagonal elements of $\mathbf{n}, \mathbf{n}^{(2)}$ contains the squares of the elements of vector $\mathbf{n}, \mathbf{p}=\left(p_{12}, p_{21}\right)^{\mathrm{T}}$ is the vector of the probabilities of switching from one sample size to another, $\mathbf{D}_{\mathbf{p}}$ is the $2 \times 2$ diagonal matrix with the diagonal elements of $\mathbf{p}$ and, finally, $\mathbf{p}^{(2)}$ contains the squares of the elements of vector $\mathbf{p}$.

At the beginning, when the process is assumed to be in-control $\left(\boldsymbol{\mu}_{1}=\boldsymbol{\mu}_{0} \& \boldsymbol{\Sigma}_{1}=\boldsymbol{\Sigma}_{0}\right), b_{1}$ and $b_{2}$ are obtained as follows:

$$
\begin{aligned}
& b_{1}=\frac{p_{11}}{p_{11}+p_{12}}, \\
& b_{2}=\frac{p_{22}}{p_{21}+p_{22}} .
\end{aligned}
$$

After computing the transition probilities via the proposed method, the general formulas in Equations (25) - (32) can be used to compute the performance measures.

\section{Numerical analyses}

In this section, for scenarios 1 and 2 defined in the previous section, by using the Matlab software, we perform numerical analyses in order to compare the adaptive schemes with each other as well as with the FP one. We perform our analyses for several number of quality characteristics $p$. We also compare our proposed scheme to one of the best existing methods. Finally, in this section, through an illustrative example we show how our scheme can be implemented in practice.

\subsection{Performance comparison of the proposed methods: Scenario-1}

First, for $p=2$, we assume that $\Sigma_{0}=\left[\begin{array}{cc}1 & 0.5 \\ 0.5 & 1\end{array}\right] \rightarrow \Sigma_{1}=\left[\begin{array}{cc}\tau & 0.5 \tau \\ 0.5 \tau & \tau\end{array}\right] ; \tau>0$. Since the in-control correlation between the quality characteristics is 0.5 and their variances are equal, then, as it was 
numerically tested as well, the following shifts $\boldsymbol{\mu}_{0}=(0,0) \rightarrow \boldsymbol{\mu}_{1}=(\delta, 0), \boldsymbol{\mu}_{0}=(0,0) \rightarrow \boldsymbol{\mu}_{1}=(0, \delta)$ and $\boldsymbol{\mu}_{0}=(0,0) \rightarrow \boldsymbol{\mu}_{1}=(\delta, \delta)$, will correspond to the same performance measures values. We also assume that $\delta \in\{0,0.1,0.3,0.7,2\}$ and $\tau \in\{1,1.05,1.2,1.5,3\}$.

For the FP scheme, assuming $n=10, \alpha=0.005$ (Equation (6) results in $U C L=3.023$ ) and $t=1$, the results for all performance measures can be seen in Table 2. In this Table, the values ANSW=0 and SDNSW=0 correspond to the FP scheme for which no switching occurs.

For the FP scheme, assuming $n=10, \alpha=0.005$ (Equation (6) results in $U C L=3.023$ ) and $t=1$, the results for all performance measures can be seen in Table 1. In this Table, the values ANSW=0 and SDNSW $=0$ correspond to the FP scheme for which no switching occurs.

For the VP scheme, assuming $A S S=10, A S I=1, A T E=0.005, \alpha_{1}=0.004, t_{2}=0.1, n_{1}=5$ and $n_{2}=15$, from Equations (11) - (16) we have: $U C L_{1}=3.0899, p_{0}=0.5, \alpha_{2}=0.006, U C L_{2}=2.9673, t_{1}=1.9$, $U W L_{1}=1.0487$ and $U W L_{2}=1.0472$. The results for all performance measures in the VP chart can be seen in Table 2, as well.

For the VSSI scheme, assuming $A S S=10, A S I=1, \alpha=0.005, t_{2}=0.1, n_{1}=5$ and $n_{2}=15$, from Equations (6), (7), (8) and (10) we have: $U C L=3.023, p_{0}=0.5, t_{1}=1.9$ and $U W L=1.0479$.

For the VSS scheme, assuming $A S S=10, t=1, \alpha=0.005, n_{1}=5$ and $n_{2}=15$, from Equations (6), (7) and (10) we have: $U C L=3.023, p_{0}=0.5$ and $U W L=1.0479$.

For the VSI scheme, assuming $A S I=1, \alpha=0.005, t_{1}=1.9, t_{2}=0.1 n=10$, from Equations (6), (8) and (10) we have: $U C L=3.023, p_{0}=0.5$ and $U W L=1.0479$.

The results for all performance measures in the VSSI, VSS and VSI charts can also be seen in Table 2. Note that, although the computations have been done for both average and standard deviation types performance measures, in order to decrease the size/number of tables, we only present in this paper the results for the average type performance measures. However, the results for standard deviation type performance measures can be easily available from the corresponding author.

"Insert Table 2 here"

From the results shown in Table 2, as the shift sizes increase, the performance measures' values decrease. Moreover, under small to moderate simultaneous and separate shifts, in terms of time to signal, VP, VSSI, VSI, VSS, and FP charts have the best to the worst performances, meaning the VP chart is the best scheme and the FP chart is the worst one. With the exception of 0.3 mean shift, in which VSS chart has a better time to signal performance than the VSI chart.

Under small to moderate simultaneous and separate shifts, in terms of the run length and number of observations to signal, VP, VSSI=VSS, VSI=FP have the best to the worst performances. In terms of the number of switches to signal, the order is FP, VP, VSSI=VSS and VSI.

Under large shifts, all the adaptive and non-adaptive charts have almost the same performances.

For $p=3$, we assume $\boldsymbol{\Sigma}_{0}=\left[\begin{array}{ccc}1 & 0.5 & 0.5 \\ 0.5 & 1 & 0.5 \\ 0.5 & 0.5 & 1\end{array}\right] \rightarrow \boldsymbol{\Sigma}_{1}=\left[\begin{array}{ccc}\tau & 0.5 \tau & 0.5 \tau \\ 0.5 \tau & \tau & 0.5 \tau \\ 0.5 \tau & 0.5 \tau & \tau\end{array}\right] ; \tau>0$. Again, as for the case $p=2$, the following shifts, $\mu_{0}=(0,0,0) \rightarrow \mu_{1}=(\delta, 0,0), \quad \mu_{0}=(0,0,0) \rightarrow \mu_{1}=(0, \delta, 0)$, $\mu_{0}=(0,0,0) \rightarrow \mu_{1}=(0,0, \delta)$ and $\mu_{0}=(0,0,0) \rightarrow \mu_{1}=(\delta, \delta, \delta)$ correspond to the same performance measures values. 
With the same methodology used for $p=2$, one can see the results for the FP, VP, VSSI, VSS and VSI charts, for $p=3$ in Table 3. All the conclusions for $p=2$ (Table 2) can be drawn for the $p=3$ case from Table 3 as well. Moreover, as it is clear from the results presented in Tables 2 and 3, as the number of quality characteristics increases, under small to relatively large mean shifts, the performance measures worsen. However, under small to large variance shifts, the charts performance improves and also, in most of the cases, under simultaneous shifts, the charts performances improve, except under 1.05 variance and 0.3 mean shifts, in which the performances deteriorate and also under 1.05 variance and 0.7 mean shifts, for which the performances only deteriorate for the FP and VSI charts.

"Insert Table 3 here"

\subsection{Performance comparison of the proposed methods: Scenario-2}

In this scenario, we assume that all the assumptions are the same as for the first scenario, except for $\Sigma_{1}$. In this scenario, we assume two cases for the shift in variance-covariance matrix.

\subsubsection{Scenario-2. Case 1: Mean and Variance Shifts}

In the first case, only the variance components increase in the variance-covariance matrix such as:

For $p=2: \Sigma_{0}=\left[\begin{array}{cc}1 & 0.5 \\ 0.5 & 1\end{array}\right] \rightarrow \boldsymbol{\Sigma}_{1}=\left[\begin{array}{cc}\tau_{1} & 0.5 \\ 0.5 & \tau_{2}\end{array}\right] ; \theta_{1}, \theta_{2} \geq 1$ and

For $p=3: \boldsymbol{\Sigma}_{0}=\left[\begin{array}{ccc}1 & 0.5 & 0.5 \\ 0.5 & 1 & 0.5 \\ 0.5 & 0.5 & 1\end{array}\right] \rightarrow \boldsymbol{\Sigma}_{1}=\left[\begin{array}{ccc}\tau_{1} & 0.5 & 0.5 \\ 0.5 & \tau_{2} & 0.5 \\ 0.5 & 0.5 & \tau_{3}\end{array}\right] ; \tau_{1}, \tau_{2}, \tau_{3} \geq 1$.

For $p=2$, we assume that:

$\tau=\left(\tau_{1}, \tau_{2}\right) \in\{(1,1),(1.05,1),(1.05,1.05),(1.05,1.3),(1.4,1.4),(3,3)\}$.

We also assume that:

$\boldsymbol{\mu}_{0}=(0,0) \rightarrow \boldsymbol{\mu}_{1}=\left(\delta_{1}, \delta_{2}\right)$ so that $\left(\delta_{1}, \delta_{2}\right) \in\{(0.0),(0.1,0),(0.1,0.3),(0.5,0.8),(2,2)$.

For $p=3$ we have:

$\tau=\left(\tau_{1}, \tau_{2}, \tau_{3}\right) \in\{(1,1,1),(1.05,1,1),(1.05,1.05,1.05),(1.05,1.3,1.05),(1.4,1.4,1.05),(3,3,1.4)\}$,

$\boldsymbol{\mu}_{0}=(0,0,0) \rightarrow \boldsymbol{\mu}_{1}=\left(\delta_{1}, \delta_{2}, \delta_{3}\right) \in\{(0,0,0),(0.1,0,0.1),(0.1,0.3,0.3),(0.5,0.8,0.5),(2,2,2)\}$.

The results for the scenario 2 case 1 for $p=2$ and 3 can be seen in Tables 4 and 5, respectively. The conclusions that have been drawn for scenario 1 (Tables 2 and 3) can be applied for this scenario as well (see Tables 4 and 5). Since in this scenario the shift sizes for $p=2$ and 3 are assumed different, there is no systematic trend in the charts' performances when the number of quality characteristics changes.

"Insert Tables 4 and 5 here"

\subsubsection{Scenario-2. Case 2: Mean and Covariance shifts}

In the second case, we assume that only the covariance component shifts in the variance-covariance matrix, such as: 
$\boldsymbol{\Sigma}_{0}=\left[\begin{array}{cc}1 & 0.5 \\ 0.5 & 1\end{array}\right] /\left[\begin{array}{ccc}1 & 0.5 & 0.5 \\ 0.5 & 1 & 0.5 \\ 0.5 & 0.5 & 1\end{array}\right] /\left[\begin{array}{cccc}1 & 0.5 & 0.5 & 0.5 \\ 0.5 & 1 & 0.5 & 0.5 \\ 0.5 & 0.5 & 1 & 0.5 \\ 0.5 & 0.5 & 0.5 & 1\end{array}\right] \rightarrow \boldsymbol{\Sigma}_{1}=\left[\begin{array}{cc}1 & \theta \\ \theta & 1\end{array}\right] /\left[\begin{array}{ccc}1 & \theta & \theta \\ \theta & 1 & \theta \\ \theta & \theta & 1\end{array}\right] /\left[\begin{array}{cccc}1 & \theta & \theta & \theta \\ \theta & 1 & \theta & \theta \\ \theta & \theta & 1 & \theta \\ \theta & \theta & \theta & 1\end{array}\right]$

$\theta \in\{0.01,0.02, \ldots, 0.99\}$, for $p=2,3$ and 4 , respectively.

For this analysis, since the trends are the same for all performance measures, in order to decrease the size of the paper we only used the most important, i.e. the ATS, performance measure in order to compare the charts. We also assume that the mean shifts for all quality characteristics are equal $\boldsymbol{\mu}_{0}=(0,0) /(0,0,0) /(0,0,0,0) \rightarrow \boldsymbol{\mu}_{1}=(\delta, \delta) /(\delta, \delta, \delta) /(\delta, \delta, \delta, \delta), \quad$ under $\quad$ none $\quad(\delta=0)$, small $(\delta=0.2)$, medium $(\delta=0.8)$ and large $(\delta=1.5)$ mean shifts. All other assumptions are as assumed similar as before. In Figures 1 to 3 , the performances of all non-adaptive and adaptive charts are compared for $p=2,3$ and 4, respectively. Since the in-control covariance is 0.5 , and the variances are assumed fixed, logically, any deviation from the in-control covariance, should result in less (better) performance measure value than the in-control one. This happens for all the charts for covariance shifts less than 0.5. However, for covariance shifts more than the in-control value (0.5), the charts' performance values increase up to some point and decreases afterward. That point, goes further away from 0.5 as the mean shifts increase and gets near to 0.5 as $p$ increases (see Figures 1 to 3 ). Moreover, in case of large mean shifts, the performances of all the charts remain almost fixed with shifts in the covariance.

"Insert Figures 1 to 3 here"

\subsection{Performance comparison of the proposed methods with the ELR Chart}

In this section we compare our proposed scheme with the one proposed by Zhang et al. (2010) which should be considered as one of the most powerful scheme available for the simultaneous monitoring of the process mean and variability as it based on the combination of the EWMA and GLR frameworks. In their paper, they compared their single-chart scheme with some available charts and they concluded that their chart surpasses them in many situations. However, as for any EWMA schemes, the choice of the optimal value for the smoothing parameter $\lambda$ is crucial for the chart to have an optimal efficiency and, unfortunately this optimal value actually depends on the size of the shift which is generally unknown. These kinds of issues do not exist in our proposed scheme. For all the charts considered for the comparison we assumed that the in-control ATS=370 and $p=2$. Also, for the FP chart we used: $\alpha=0.0027$ (to have the in-control ATS=370), $n=5, t=1$, for the VP chart we used: $A S S=5, A S I=1, A T E=0.0027$ (to have the in-control ATS=370), $\alpha_{1}=0.0017, t_{2}=0.1, n_{1}=3, n_{1}=7$. The ATS for the ELR scheme is also obtained with $n=5, t=1$ and for different $\lambda$ values $(0.1,0.2,0.3$ and 0.4 ). In addition, the parameters used in Table 5 to represent the shifts are:

$\boldsymbol{\mu}_{0}=(0,0) \rightarrow \boldsymbol{\mu}_{1}=\left(\delta_{1}, \delta_{2}\right)$ and $\boldsymbol{\Sigma}_{0}=\left[\begin{array}{ll}1 & 0 \\ 0 & 1\end{array}\right] \rightarrow \boldsymbol{\Sigma}_{1}=\left[\begin{array}{cc}\tau_{1} & \rho \sqrt{\tau_{1} \tau_{2}} \\ \rho \sqrt{\tau_{1} \tau_{2}} & \tau_{2}\end{array}\right]$.

Moreover, the performance of the ELR chart has been obtained based on 10000 simulation runs. We used 30 different separate as well as simultaneous shifts and, as it can be easily seen in Table 6, in many situations the proposed scheme surpasses the ELR chart. More precisely, among the 30 situations investigated, the scheme proposed in this paper is better than the ELR chart in 16 cases, i.e. 
about $50 \%$ of the time. However, unlike the ELR scheme (as explained above), our scheme can be easily implemented in practice.

\section{"Insert Tables 6 here"}

\subsection{An illustrative example}

For illustration of the proposed adaptive method, and to show how it works, the illustrative example presented by Aparisi (1996) is adopted. We have a mechanical part with $p=3$ main quality characteristics as it is shown in Figure 4. The quality characteristics are the width $X_{1}$, the inner diameter $X_{2}$ and the length $X_{3}$.

\section{"Insert Figure 4 here"}

Based on Aparisi (1996)'s assumptions, the average Type-I error is ATE $=0.005$ and the mean vector and the variance-covariance matrix of the quality characteristics are:

$$
\boldsymbol{\mu}_{0}=(7,3,15)^{\prime}, \boldsymbol{\Sigma}_{0}=\left[\begin{array}{ccc}
0.2 & 0.054 & 0.162 \\
0.054 & 0.09 & 0.042 \\
0.162 & 0.042 & 0.31
\end{array}\right] \text {. }
$$

Since it was proven in the previous sections that the VP scheme has the best performance, we adopt this scheme for our process monitoring. We use the VP strategy explained in section 3 and all the other chart parameters values related to the adaptive scheme are assumed as (to be determined by quality experts): $A S S=10, A S I=1 h r, \alpha_{1}=0.004, t_{2}=0.1, n_{1}=5$ and $n_{2}=15$. Having the assumed parameters values, by using Equation (11) we obtain $U C L_{1}=3.0899$, by using Equation (14) we obtain $U C L_{2}=2.9673$, by using Equation (15) we obtain $t_{1}=1.9 \mathrm{hrs}$, and at last, by using Equation (16) we obtain $U W L_{1}=1.0487$ and $U W L_{2}=1.0472$.

For the second to the last columns of Table 7, respectively we have: the number of samples taken for the current sample, the cumulative number of samples taken up to the current sample, the sampling interval adopted for reaching the current sample, the cumulative sampling intervals up to the current sample, the total number of switches between the in-control states up to the current sample, the $M_{i}$, $V_{i}$ and $C_{i}$ statistics values for the current sample (which are computed by using Equations (3), (4) and (5), respectively), $U W L$ and $U C L$ adopted for the current sample, and finally, the state of the process after taking the current sample.

Before beginning the process monitoring, we randomly shift the mean and variability as:

$$
\boldsymbol{\mu}_{1}=(7.05,3.01,15)^{\prime}, \boldsymbol{\Sigma}_{1}=\left[\begin{array}{ccc}
0.2 & 0.0545 & 0.162 \\
0.0545 & 0.095 & 0.041 \\
0.162 & 0.041 & 0.31
\end{array}\right] \text {. }
$$

We have generated 20 consecutive samples for the process monitoring. As it can be seen in Table 7, the proposed VP chart was able to find an out-of-control situation in sample \#17; after 10.6 hours, 205 samples measured and with three shifts between in-control states. After finding an out-of-control situation, quality practitioners should investigate the cause and find the problem source.

\section{"Insert Table 7 here"}

In addition, the proposed VP control chart can also be seen graphically (plotted using MS Excel) in Figure 5.

\section{"Insert Figure 5 here"}




\section{Conclusions and future research}

A new multivariate control chart based on an already existing bivariate chart has been introduced in order to monitor both process parameters (mean and variation) simultaneously and by using only a single max-type chart. After establishing the multivariate control chart, adaptive schemes have been proposed to make the chart's performance better in detecting small and moderate shifts. In order to measure the charts' performances and to compare the non-adaptive with the adaptive charts, eight performance measures have been used, each measuring one aspect of the performance. The average run length and its standard deviation, the average time to signal its standard deviation, the average number of observations to signal and its standard deviation, the average number of switches to signal and its standard deviation are the performance measures used. In order to compute these performance measures, a new Markov chain model has been proposed with the derivation of the corresponding transition probabilities. We used two scenarios for the variance-covariance matrix shifts. In the first scenario, the shifted variance-covariance matrix is a constant multiplier of the in-control one. In the second scenario, the more general one, the elements in the variance-covariance matrix are allowed to change independently. We also considered two cases: in the first, only the variances shift but the covariance components stay unchanged. However, in the second case, only the covariance components shift. Through extensive numerical analysis, adaptive schemes were found to perform much better under small to moderate separate and simultaneous shifts in all scenarios; VP and VSSI charts, respectively, having the best performances. However, the adaptive and FP charts almost have the same performance under large shifts. In the case two of the second scenario, in which only the covariance shifts and the variances remain fixed, we concluded that an increasing shift in the covariance components, would result in negative performance of all the charts up to some point (covariance value). However, that point gets closer to the in-control value as the shift in the mean decreases (except for large mean shifts in which the chart performance is almost insensible to the covariance change) or, also, as the number of quality characteristics increases. Moreover, our results showed that in the first scenario, in which the variance and covariance components shift with an equal multiplier, with the increase in the number of quality characteristics, as the mean shift increases to a relatively large one, the performance measures' values increase. However, as the variability shift increases and also in most simultaneous mean and variability shifts, the performance measures improve. In addition, we compared the proposed scheme with the ELR scheme and the results showed that our scheme performs at least as good as the ELR method and can be easily implemented in practice, unlike the ELR scheme. Finally, through a numerical example, the implementation of the proposed adaptive scheme was shown in practice.

Concerning possible extensions of this paper, researchers can apply the proposed adaptive schemes and also the proposed Markov chain model for other types simultaneous control charts, especially max-type ones. Moreover, the effect of estimated parameters, measurement errors as well as autocorrelation can be considered on the performance of the proposed control charts as future research. 


\section{References}

Alt, F. B. (1985). Multivariate control charts. Encyclopedia of Statistical Sciences 6. Kotz, S., Johnson, N. L., Eds.; Wiley, 110-122.

Aparisi, F. (1996). Hotelling's T ${ }^{2}$ control chart with adaptive sample sizes. International Journal of Production Research, 34(10), 2853-2862.

Aparisi, F., Jabaloyes, J. \& Carrion, A. (1999). Statistical properties of the $|\mathrm{S}|$ multivariate control chart. Communications in Statistics - Theory and Methods, 28(11), 2671-2686.

Aparisi, F., Jabaloyes, J., \& Carrion, A. (2001). Generalized variance chart design with adaptive sample sizes. The bivariate case. Communications in Statistics - Simulation and Computation, 30(4), 931-948.

Aparisi, F., \& Haro, C. L. (2001). Hotelling's $\mathrm{T}^{2}$ control chart with variable sampling intervals. International Journal of Production Research, 39(14), 3127-3140.

Aparisi, F., \& Haro, C. L. (2003). A comparison of $\mathrm{T}^{2}$ charts with variable sampling scheme as opposed to MEWMA. International Journal of Production Research, 41(10), 2169-2182.

Chen, G., Cheng, S. W., \& Xie, H. (2005). A new multivariate control chart for monitoring both location and dispersion. Communications in Statistics-Simulation and Computation, 34(1), 203-217.

Chen, Y. K. (2007). Adaptive sampling enhancement of Hotelling's $\mathrm{T}^{2}$ control charts. European Journal of Operational Research, 178 (3), 841-857.

Chong N.L., Khoo M.B.C, Haq A. and Castagliola P. (2019). Hotelling's $T^{2}$ control charts with fixed and variable sample sizes for monitoring short production runs. Quality and Reliability Engineering Internationa, 35(1), 14-29.

Costa, A. F. B., \& Faria Neto, A. (2017). The S chart with variable charting statistic to control bi and trivariate processes. Computers \& Industrial Engineering, 113, 27-34.

Faraz, A., \& Parsian, A. (2006). Hotelling's $\mathrm{T}^{2}$ control chart with double warning lines. Statistical Papers, 47(4), 569-693.

Faraz, A., \& Moghadam, M. B. (2009). Hotelling's $\mathrm{T}^{2}$ control chart with two-state adaptive sample size. Quality \& Quantity, 43(6), 903-913.

Faraz, A., Chalaki, K., \& Moghadam, M.B. (2011). On the properties of the Hotelling's $T^{2}$ control chart with VSI scheme. Quality \& Quantity, 45(3), 579-586.

Faraz, A., Heuchenne, C., Saniga, E., \& Costa, A. F. B. (2014). Double-objective economic statistical design of the VP $\mathrm{T}^{2}$ control chart: Wald's Identity approach. Journal of Statistical Computation and Simulation, 84(10), 2123-2137.

Gnanadesikan, M., \& Gupta, S. S. (1970). A selection procedure for multivariate normal distributions in terms of the generalized variances. Technometrics, 12, 103-117.

Grigoryan, A., \& He, D. (2005). Multivariate double sampling $|S|$ charts for controlling process variability. International Journal of Production Research, 43(4), 715-730.

Hawkins, D. M., \& Maboudou-Tchao, E. M. (2008). Multivariate exponentially weighted moving covariance matrix. Technometrics, 50(1), 155-166.

Hotelling, H. (1947). Multivariate quality control - Illustrated by the air testing of sample bombsights. In: Eisenhart, C., M.W. Hastay, and W.A. Wallis, editors. Techniques of Statistical Analysis, NewYork: McGraw-Hill, 111-184.

Jensen, W. A., Bryce, G. R., \& Reynolds Jr., M. R. (2008). Design issues for adaptive control charts. Quality and Reliability Engineering International, 24(4), 429-445.

Khoo, M. B. C. (2005). A new bivariate control chart to monitor the multivariate process mean and variance simultaneously. Quality Engineering, 17, 109-118.

Khoo, M. B. C., Wu, Z., Castagliola, P., \& Lee, H. C. (2013). A multivariate synthetic double sampling T ${ }^{2}$ control chart. Computers \& Industrial Engineering, 64, 179-189. 
Lee, M. H., \& Khoo, M. B. C. (2015). Multivariate synthetic $|S|$ control chart with variable sampling interval. Communications in Statistics - Simulation and Computation, 44(4), 924-942.

Reynolds Jr., M. R., \& Cho, G.Y. (2006). Multivariate control charts for monitoring the mean vector and covariance matrix. Journal of Quality Technology, 38(3), 230-253.

Reynolds Jr., M. R., \& Kim, K. (2007). Multivariate control charts for monitoring the process mean and variability using sequential sampling. Sequential Analysis: Design Methods and Applications, 26, $283-$ 315.

Reynolds Jr., M. R., \& Cho, G.Y. (2011). Multivariate control charts for monitoring the mean vector and covariance matrix with variable sampling intervals. Sequential Analysis: Design Methods and Applications, 30, 1-40.

Sabahno, H., Amiri, A., \& Castagliola, P. (2018). Evaluating the effect of measurement errors on the variable sampling intervals Hotelling $\mathrm{T}^{2}$ control charts. Quality and Reliability Engineering International, 34(8), 1785-1799.

Sabahno, H., Amiri, A., \& Castagliola, P. (2019). Optimal performance of the variable sample sizes Hotelling's $\mathrm{T}^{2}$ control chart in the presence of measurement errors. Quality Technology \& Quantitative Management, 16(5), 588-612.

Seif, A., Faraz, A., Saniga, E., \& Heuchenne, C. (2016). A statistically adaptive sampling policy to the Hotelling's $\mathrm{T}^{2}$ control chart: Markov Chain approach. Communications in Statistics-Theory and Methods, 45(13), 3919-3919.

Du, S., Lv, J., \& Xi, L. (2012). On-line classifying process mean shifts in multivariate control charts based on multiclass support vector machines. International Journal of Production Research, 50(22), 6288-6310.

Wang, K., Yeh, A. B., \& Li, B. (2014). Simultaneous monitoring of process mean vector and covariance matrix via penalized likelihood estimation. Computational Statistics \& Data Analysis, 78(1), 206-217.

Yeh, A., \& Lin., D. (2002). A new variables control chart for simultaneously monitoring multivariate process mean and variability. International Journal of Reliability, Quality and Safety Engineering, 91, 41-59.

Yeh, A., Lin, D., Zhou, H., \& Venkataramani, C. (2003). A multivariate exponentially weighted moving average control chart for monitoring process variability. Journal of Applied Statistics, 30(5), 507-536.

Zhang, G., and Chang, S. I. (2008). Multivariate EWMA control charts using individual observations for process mean and variance monitoring and diagnosis. International Journal of Production Research, 46(1), 6855-6881.

Zhang, J., Li, Z., \& Wang, Z. (2010). A multivariate control chart for simultaneously monitoring process mean and variability. Computational Statistics \& Data Analysis, 54(10), 2244-2252. 
Table 1 Literature review in comparison with our paper

\begin{tabular}{|c|c|c|c|c|c|}
\hline Paper & Control chart type & Variables & Adaptive & Simultaneous & $\begin{array}{l}\text { Computation method of } \\
\text { performance measures }\end{array}$ \\
\hline Aparisi (1996) & VSS Hotelling's T $^{2}$ & Multivariate & $\checkmark$ & - & Markov chains \\
\hline Aparisi et al. (1999) & $|S|$ & Multivariate & - & - & Probabilistic relations \\
\hline Aparisi and Haro (2001) & VSI Hotelling's T $^{2}$ & Multivariate & $\checkmark$ & - & Markov chains \\
\hline Aparisi et al. (2001) & VSS $|S|$ & Bivariate & $\checkmark$ & - & Markov chains \\
\hline Yeh and Lin (2002) & Box & Multivariate & - & $\checkmark$ & Monte Carlo simulation \\
\hline Yeh et al. (2003) & EWMA-V , EWMA-M & Multivariate & - & $\checkmark$ & Monte Carlo simulation \\
\hline Aparisi and Haro (2003) & VSSI Hotelling's T² & Multivariate & $\checkmark$ & - & Markov chains \\
\hline Khoo (2005) & a Max-Type Chart & Bivariate & - & $\checkmark$ & Monte Carlo simulation \\
\hline Grigoryan and $\mathrm{He}(2005)$ & $\operatorname{MDS}|S|$ & Multivariate & $\checkmark$ & - & Monte Carlo simulation \\
\hline Chen et al. (2005) & Max-MEWMA & Multivariate & - & $\checkmark$ & Monte Carlo simulation \\
\hline Reynolds and Cho (2006) & MEWMA-SSDM & Multivariate & - & $\checkmark$ & Monte Carlo simulation \\
\hline Faraz and Parsian (2006) & VSSI-DWL Hotelling T $^{2}$ & Multivariate & $\checkmark$ & - & Markov chains \\
\hline Chen (2007) & VP Hotelling T $^{2}$ & Multivariate & $\checkmark$ & - & Probabilistic relations \\
\hline Reynolds and Kim (2007) & SS Shewharts\&MEWMAs & Multivariate & $\checkmark$ & $\checkmark$ & Monte Carlo simulation \\
\hline Hawkins and Maboudou-Tchao (2008) & MEWMA-MEWMC & Multivariate & - & $\checkmark$ & Monte Carlo simulation \\
\hline Zhang and Chang (2008) & two MEWMAs (individual observations) & Multivariate & - & $\checkmark$ & Monte Carlo simulation \\
\hline Faraz and Moghadam (2009) & VSS Hotelling's T $^{2}$ & Multivariate & $\checkmark$ & - & Markov chains \\
\hline Zhang et al. (2010) & ELR & Multivariate & - & $\checkmark$ & Monte Carlo simulation \\
\hline Faraz et al. (2011) & VSI Hotelling's T $^{2}$ & Multivariate & $\checkmark$ & - & Markov chains \\
\hline Reynolds and Cho (2011) & VSI-SS Shewharts\&MEWMAs & Multivariate & $\checkmark$ & $\checkmark$ & Monte Carlo simulation \\
\hline Khoo et al. (2013) & Synthetic Double Sampling Hotelling's T ${ }^{2}$ & Multivariate & $\checkmark$ & - & Markov chains \\
\hline Faraz et al. (2014) & VP Hotelling's T $^{2}$ & Multivariate & $\checkmark$ & - & Probabilistic relations \\
\hline Wang et al. (2014) & pGLR-pMEWMAC (individual observations) & Multivariate & - & $\checkmark$ & Monte Carlo simulation \\
\hline Lee and Khoo (2015) & VSI Synthetic $|S|$ & Multivariate & $\checkmark$ & - & Probabilistic relations \\
\hline Seif et al. (2016) & VP-VSSCL-VSICL Hotelling T ${ }^{2}$ & Multivariate & $\checkmark$ & - & Markov chains \\
\hline Costa and Neto (2017) & VCS S & Trivariate & $\checkmark$ & - & Probabilistic relations \\
\hline Sabahno et al. (2018) & VSI Hotelling's $\mathrm{T}^{2}$ (with measurement errors) & Multivariate & $\checkmark$ & - & Markov chains \\
\hline Sabahno et al. (2019) & VSS Hotelling's T²(with measurement errors) & Multivariate & $\checkmark$ & - & Markov chains \\
\hline Chong et al. (2019) & VSS Hotelling $\mathrm{T}^{2}$ (with finite horizon) & Multivariate & $\checkmark$ & - & Markov chains \\
\hline Current Paper & a new VP / VSS / VSS / VSI Max Type Chart & Multivariate & $\checkmark$ & $\checkmark$ & a new Markov chain model \\
\hline
\end{tabular}




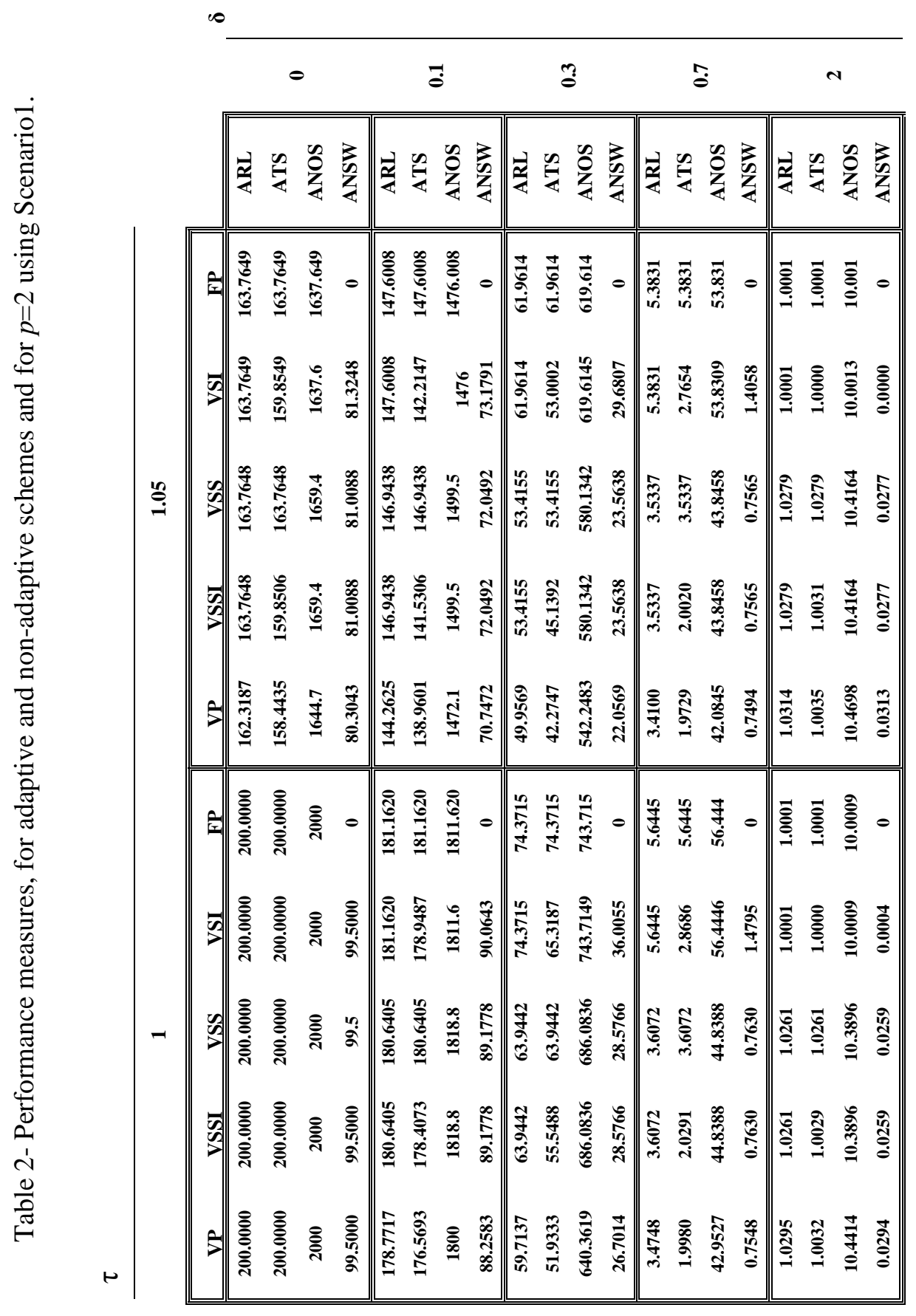




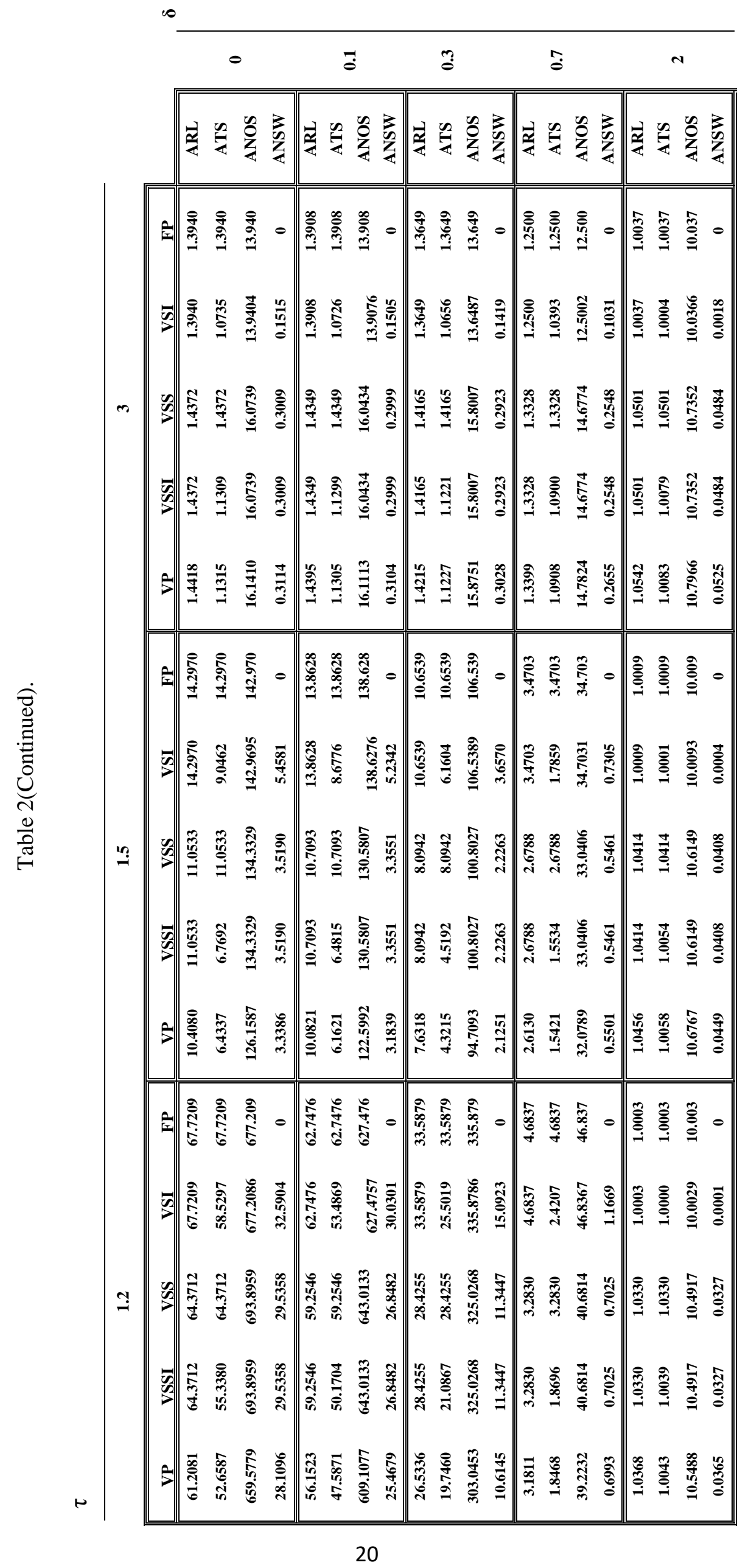




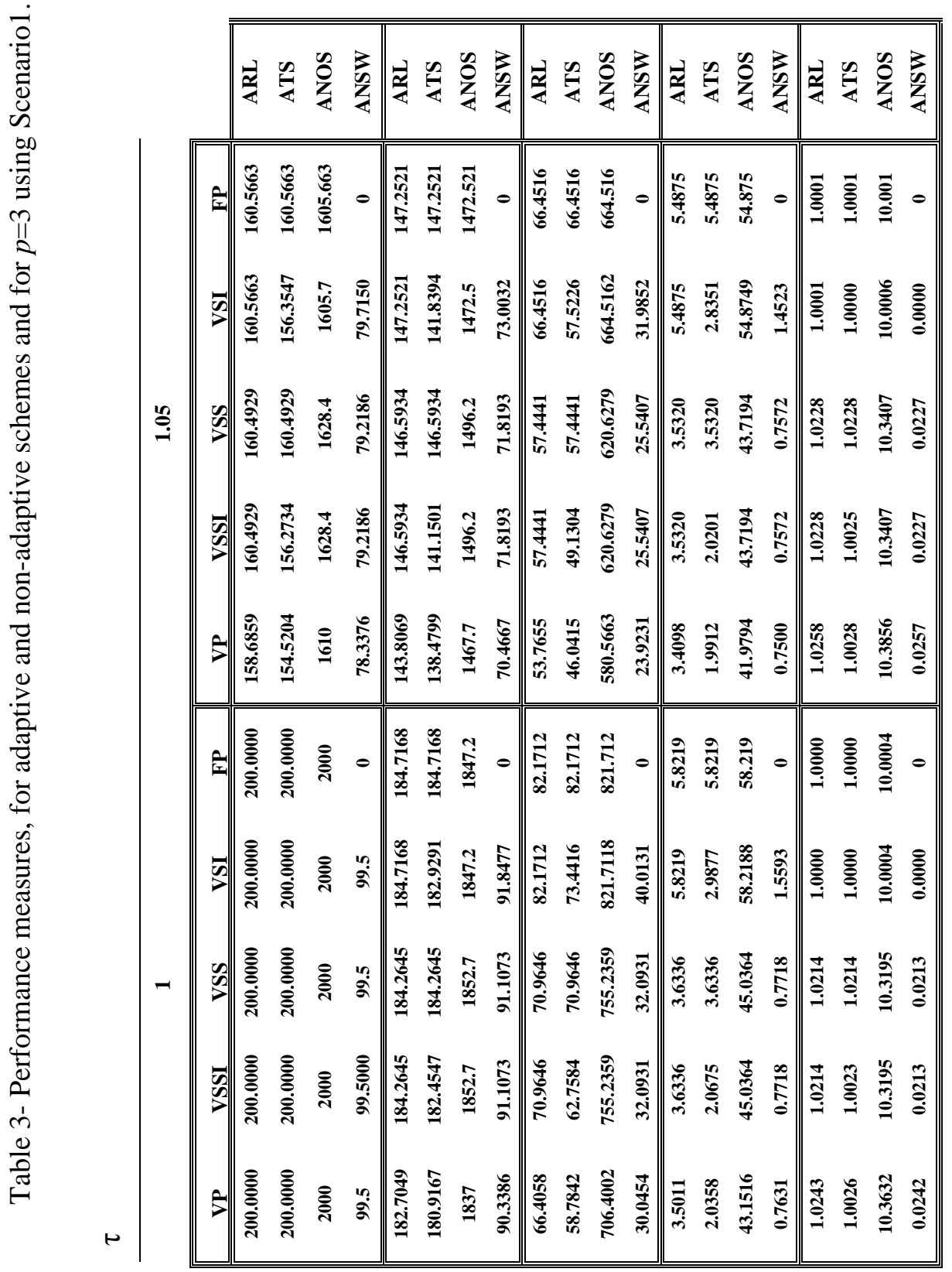




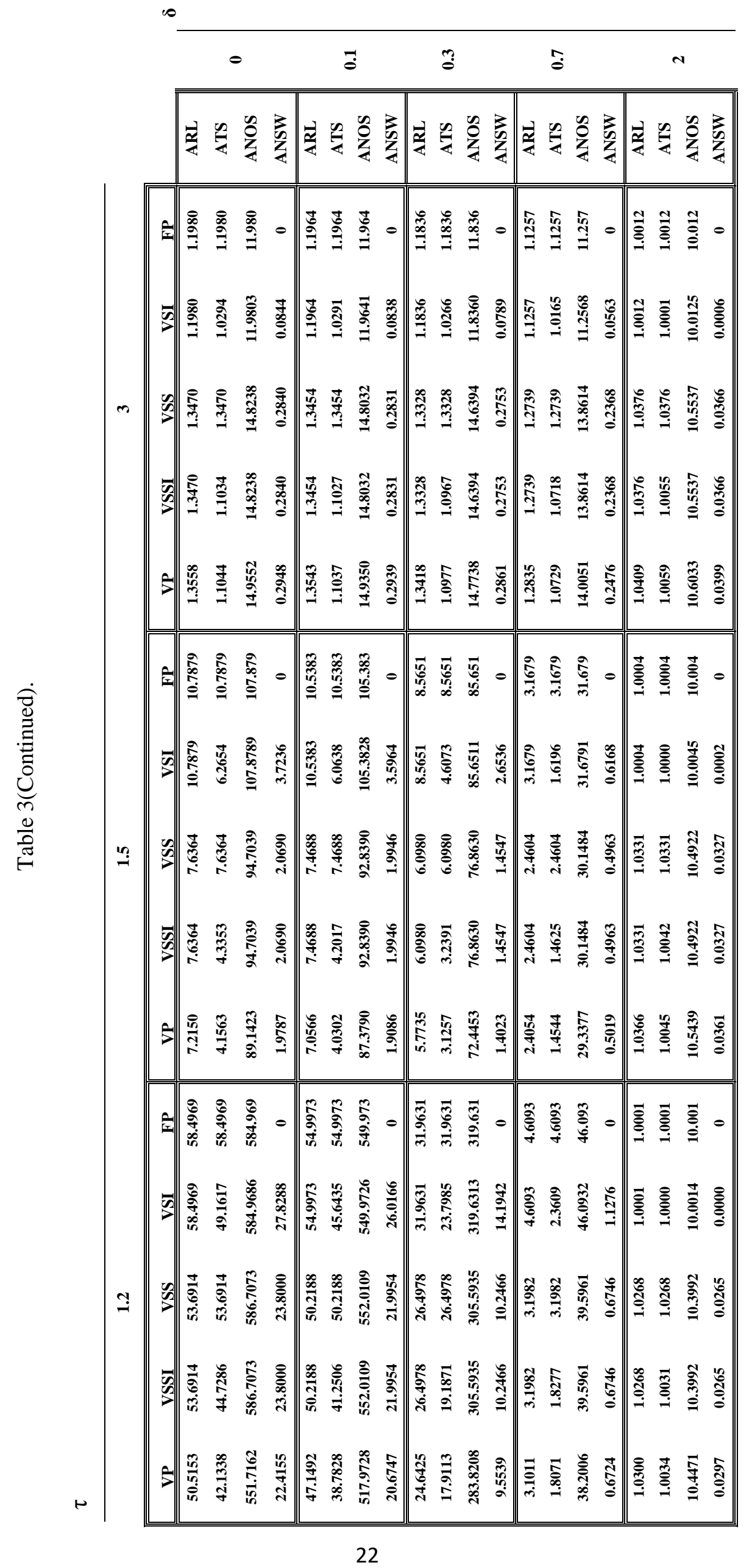




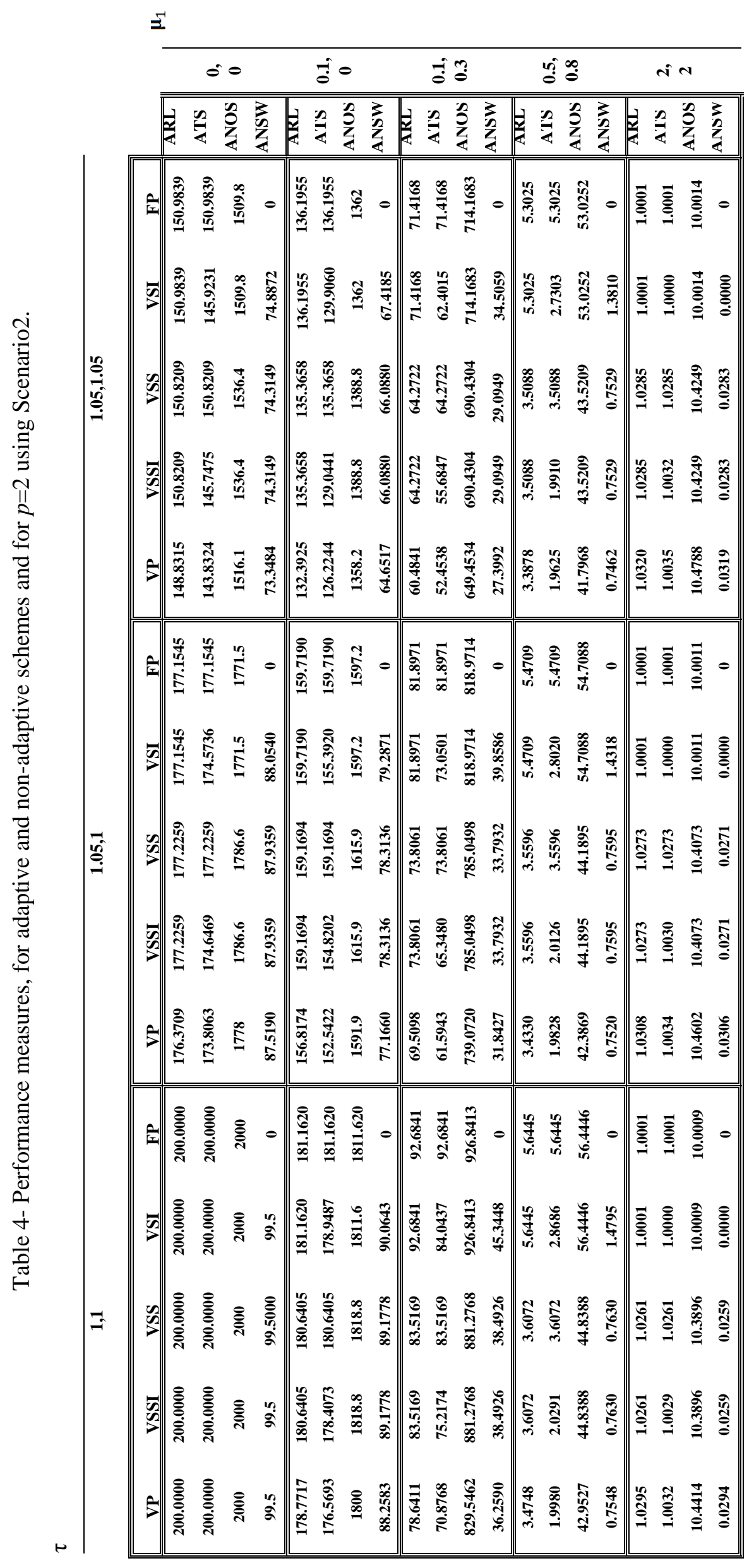




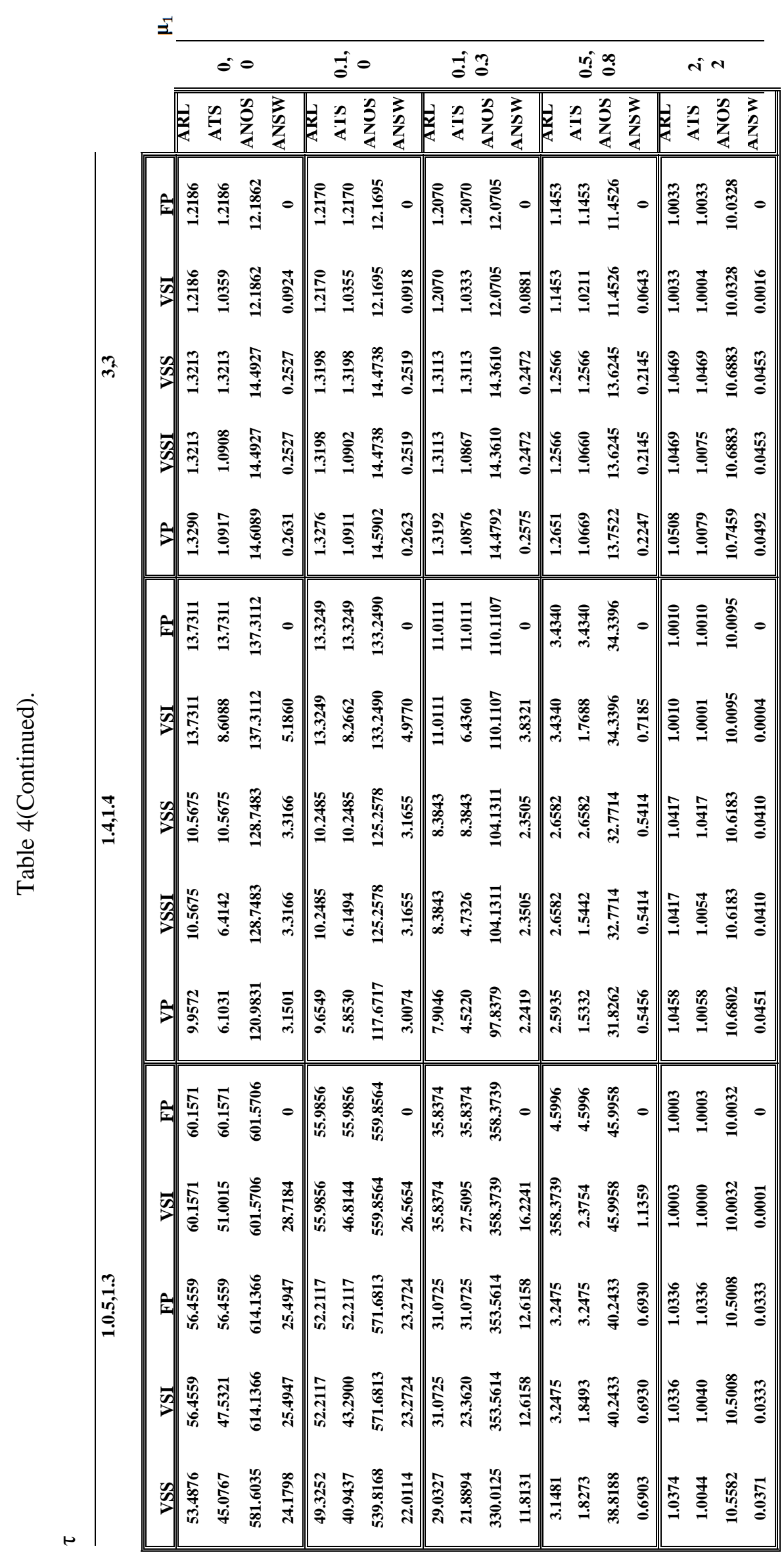




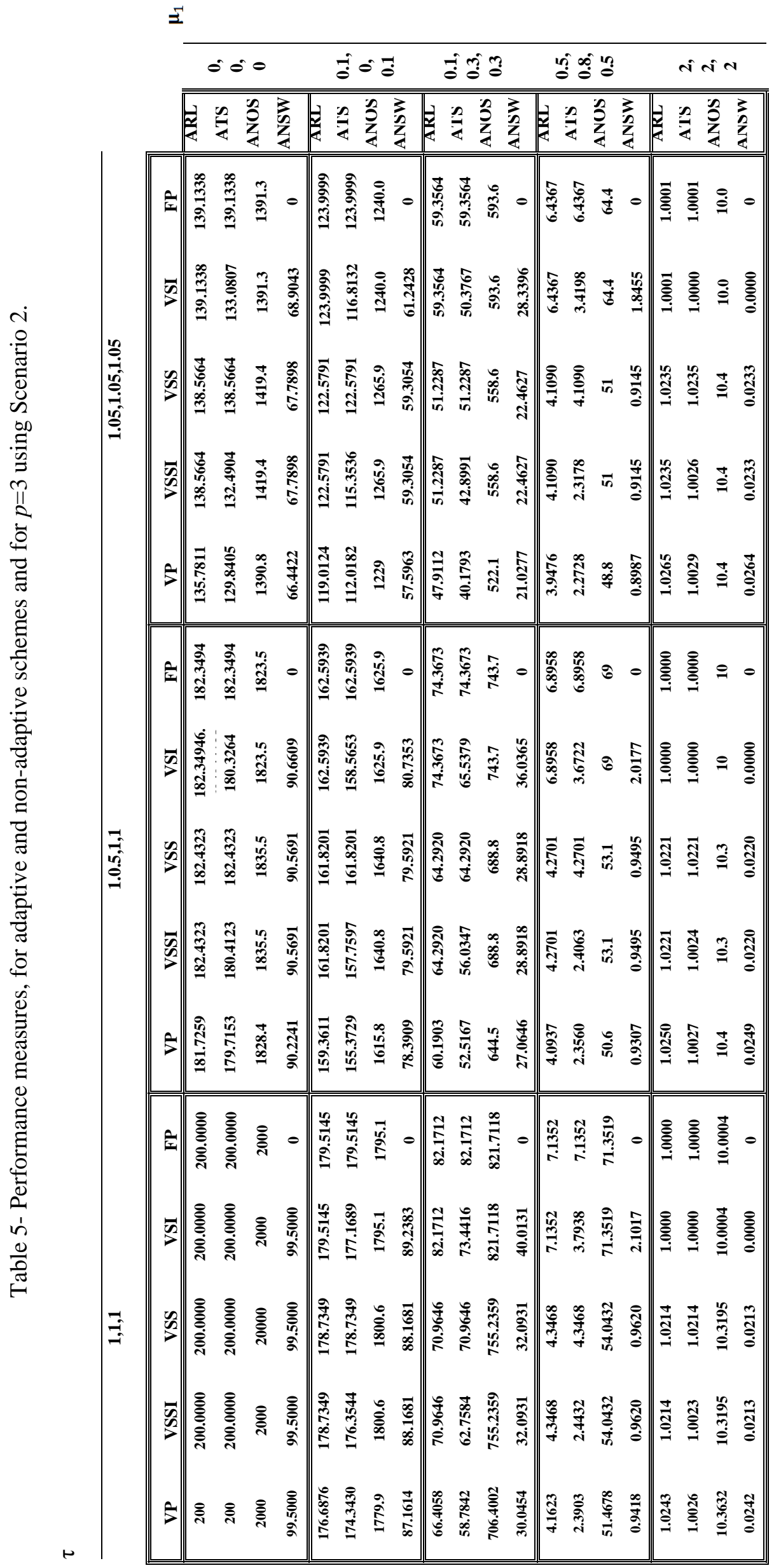




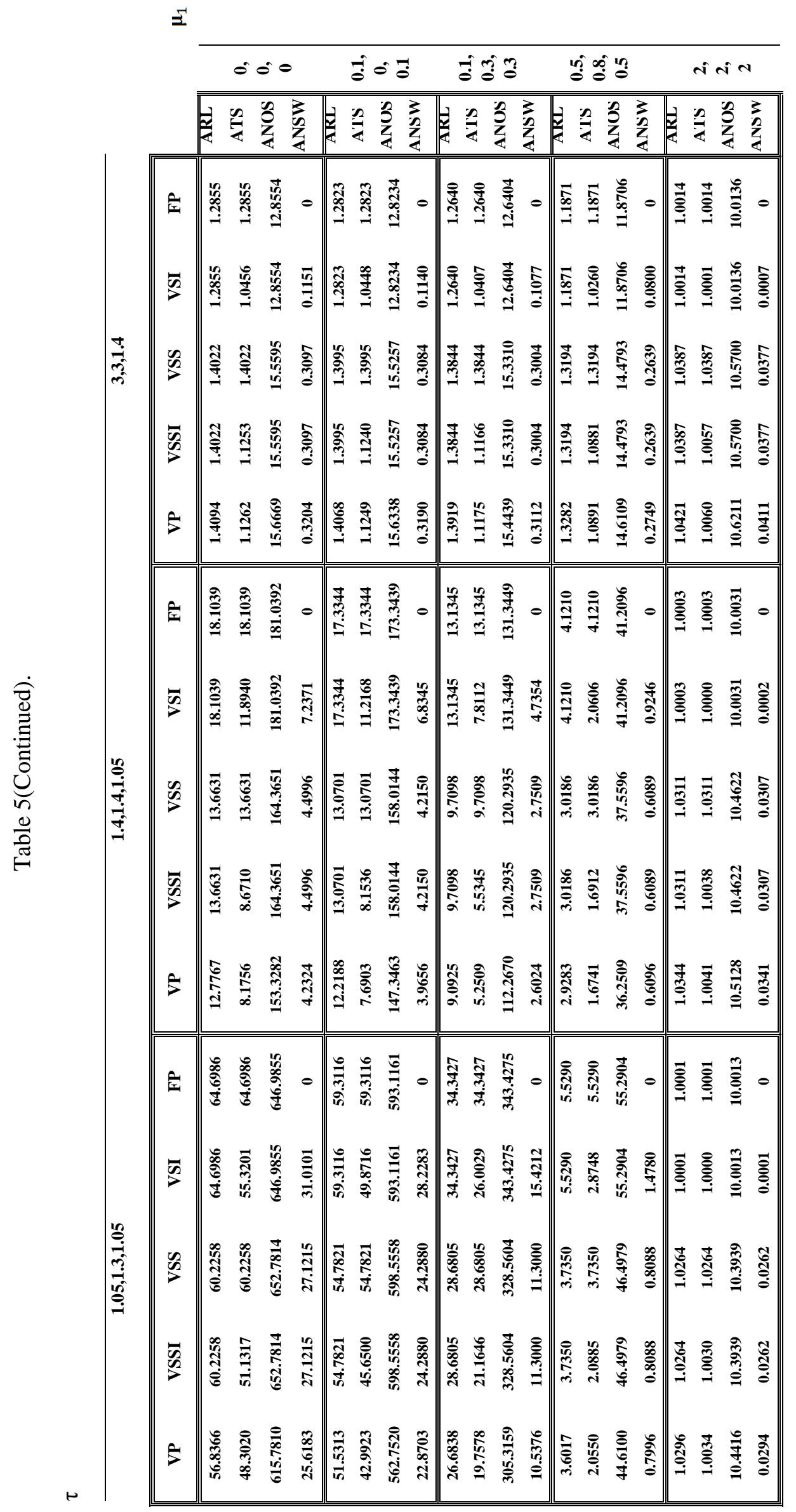


Table 6 ATS comparison of the proposed schemes versus the ELR scheme when $p=2, A S S=5, A S I=1$ and incontrol ATS=370.

\begin{tabular}{|c|c|c|c|c|c|c|}
\hline \multirow[t]{2}{*}{$\left(\delta_{1}, \delta_{2}, \sqrt{\tau_{1}}, \sqrt{\tau_{1}}, \rho\right)$} & \multicolumn{4}{|c|}{ ELR } & \multirow[b]{2}{*}{$\mathrm{FP}$} & \multirow[b]{2}{*}{$\mathrm{VP}$} \\
\hline & $\lambda=0.1$ & $\lambda=0.2$ & $\lambda=0.3$ & $\lambda=0.4$ & & \\
\hline$(0.2,0.2,1,1,0)$ & $\underline{\underline{32.1}}$ & 42.5 & "59.3 & 777.0 & 260.4 & 232.9 \\
\hline$(0.5,0.5,1,1,0)$ & 7.6 & $\underline{7.1}$ & 7.3 & 8.2 & 44.8 & 20.6 \\
\hline$(0.8,0,1,1,0)$ & 6.5 & $\underline{5.9}$ & $\underline{5.9}$ & 6.2 & 29.2 & 11.3 \\
\hline$(0,0,1.2,1.2,0)$ & $\underline{\mathbf{1 7 . 5}}$ & 21.6 & 27.2 & 33.5 & 51.5 & 32.3 \\
\hline$(0,7,0,7,1,1,0)$ & 5.0 & 4.3 & 4.2 & 4.2 & 13.0 & $\underline{3.9}$ \\
\hline$(0,0,1.5,1,0)$ & $\underline{7.6}$ & $\underline{7.6}$ & 8.4 & 9.1 & 41.1 & 24.0 \\
\hline$(0.2,0.2,1.5,1,0)$ & 6.8 & $\underline{6.6}$ & 7.0 & 7.9 & 33.7 & 18.5 \\
\hline$(0.5,0,1.5,1,0)$ & 5.6 & $\underline{5.3}$ & 5.4 & 5.7 & 22.7 & 10.9 \\
\hline$(0.5,0.2,1.5,1.2,0)$ & 5.0 & 4.6 & 4.7 & 4.9 & 10.8 & $\underline{4.3}$ \\
\hline$(1.1,1.1,1,1,0)$ & 2.8 & 2.4 & 2.2 & 2.1 & 2.4 & $\underline{1.2}$ \\
\hline$(0,0,1.5,1.5,0)$ & 4.7 & 4.2 & 4.3 & 4.4 & 6.9 & $\underline{2.7}$ \\
\hline$(0.2,0.2,1.5,1.5,0)$ & 4.4 & 3.9 & 3.9 & 4.2 & 6.5 & $\underline{2.5}$ \\
\hline$(1.5,1,1,1,0)$ & 2.3 & 2.0 & 1.9 & 1.8 & 1.6 & $\underline{1.1}$ \\
\hline$(0,0,1.75,1.75,0)$ & 2.8 & 2.5 & 2.4 & 2.3 & 3.0 & $\underline{1.5}$ \\
\hline$(1.5,1.5,1,1,0)$ & 1.9 & 1.7 & 1.5 & 1.5 & 1.2 & $\underline{1.0}$ \\
\hline$(2,2,1,1,0)$ & 1.3 & 1.1 & 1.1 & $\underline{1}$ & $\underline{1}$ & $\underline{1}$ \\
\hline$(0,0,1.5,1,0.2)$ & 7.3 & 7.0 & 7.6 & 8.2 & 7.1 & $\underline{2.8}$ \\
\hline$(0.2,0,1.5,1,0.2)$ & 6.9 & $\underline{6.7}$ & 7 & 7.5 & 41.4 & 24.3 \\
\hline$(0.2,0.2,1.5,1,0.2)$ & 6.5 & $\underline{6.2}$ & 6.6 & 7.1 & 37.3 & 21.2 \\
\hline$(0.5,0,1.5,1,0.2)$ & 5.5 & $\underline{5.1}$ & $\underline{5.1}$ & 5.5 & 24.6 & 12.1 \\
\hline$(0.5,0.5,1.5,1,0.2)$ & 4.6 & $\underline{4.1}$ & $\underline{4.1}$ & $\underline{4.1}$ & 14.5 & 6.0 \\
\hline$(0.5,0.2,1.5,1.2,0.2)$ & 5.0 & $\underline{4.5}$ & $\underline{4.5}$ & 4.7 & 11.6 & 4.7 \\
\hline$(0,0,1.5,1.5,0.2)$ & 4.5 & 4.1 & 4.2 & 4.3 & 7.4 & $\underline{2.9}$ \\
\hline$(0.2,0,1.5,1.5,0.2)$ & 4.4 & 3.9 & 4.0 & 4.1 & 7.2 & $\underline{2.8}$ \\
\hline$(0.2,0.2,1.5,1.5,0.2)$ & 4.3 & 3.9 & 3.9 & 4.0 & 6.9 & $\underline{2.7}$ \\
\hline$(0.5,0,1.5,1.5,0.2)$ & 3.9 & 3.5 & 3.4 & 3.4 & 5.9 & $\underline{2.3}$ \\
\hline$(0.5,0.5,1.5,1.5,0.2)$ & 3.5 & 3.1 & 3.0 & 3.0 & 4.9 & $\underline{2.0}$ \\
\hline$(0.5,0.5,1.5,1,0.5)$ & 4.1 & 3.6 & 3.6 & $\underline{3.5}$ & 20.9 & 9.1 \\
\hline$(0.2,0.2,1.5,1.5,0.5)$ & 3.8 & $\underline{3.4}$ & $\underline{3.4}$ & $\underline{3.4}$ & 10.7 & 4.3 \\
\hline$(0.5,0.5,1.5,1.5,0.5)$ & 3.2 & 2.8 & 2.7 & 2.7 & 6.6 & $\underline{2.6}$ \\
\hline
\end{tabular}


Table 7- Details of the first 20 samples taken for the illustrative example

\begin{tabular}{|c|c|c|c|c|c|c|c|c|c|c|c|}
\hline$i$ & $n_{i}$ & $\sum n_{i}$ & $t_{i}$ & $\sum t_{i}$ & $\begin{array}{c}\text { Sum of } \\
\text { Switches } \\
\end{array}$ & $M_{i}$ & $V_{i}$ & $C_{i}$ & $U W L_{i}$ & $U C L_{i}$ & Status \\
\hline 1 & 5 & 5 & 1.9 & 1.9 & $\begin{array}{l}0 \\
\end{array}$ & 0.1318 & 0.0732 & 0.1318 & 1.0487 & 3.0899 & In-control \\
\hline 2 & (25 & 10 & 1.9 & 3.8 & 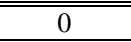 & -0.0463 & -0.0322 & 年 0.0463 & 1.0487 & 3.0899 & In-control \\
\hline $\begin{array}{l}3 \\
\end{array}$ & 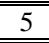 & 15 & 1.9 & 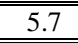 & "0 & 1.1533 & " 0.5718 & 1.1533 & " 1.0487 & $\begin{array}{l}3.0899 \\
\end{array}$ & In-control \\
\hline $\begin{array}{l}4 \\
\end{array}$ & 15 & 30 & $\begin{array}{ll}0.1 \\
\end{array}$ & "5.8 & $\begin{array}{ll}1 \\
\end{array}$ & "1.2119 & -0.0515 & "1.2119 & $\begin{array}{l}1.0472 \\
\end{array}$ & 2.9673 & In-control \\
\hline 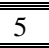 & 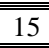 & 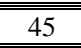 & 0.1 & 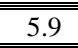 & $\begin{array}{ll}1 \\
\end{array}$ & 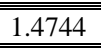 & 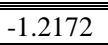 & 101.4744 & 101.0472 & 2.9673 & In-control \\
\hline 6 & 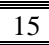 & 60 & 0.1 & 6 & 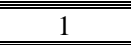 & (20.1525 & $\begin{array}{l}0.0259 \\
\end{array}$ & 0.1525 & $\begin{array}{ll}1.0472 \\
\end{array}$ & 2.9673 & $\begin{array}{l}\text { In-control } \\
\end{array}$ \\
\hline 7 & 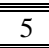 & 65 & 1.9 & 7.9 & 2 & 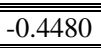 & $\begin{array}{l}0.4541 \\
\end{array}$ & $\begin{array}{l}0.4480 \\
\end{array}$ & $\begin{array}{l}1.0487 \\
\end{array}$ & $\begin{array}{l}3.0899 \\
\end{array}$ & I"In-control \\
\hline 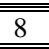 & 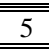 & 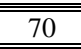 & 1.9 & 9.7 & 2 & 0.8321 & 1.4539 & 1.4539 & $\begin{array}{l}1.0487 \\
\end{array}$ & $\begin{array}{l}3.0899 \\
\end{array}$ & I"In-control \\
\hline $\begin{array}{l}9 \\
\end{array}$ & 15 & 85 & 0.1 & 9.8 & $\begin{array}{l}3 \\
\end{array}$ & $\begin{array}{l}1.1209 \\
\end{array}$ & 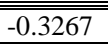 & 1.1209 & 1.0472 & 2.9673 & In-control \\
\hline 10 & 15 & 100 & 0.1 & 9.9 & 3 & 2.5332 & 至 0.7967 & 2.5332 & 1.0472 & 2.9673 & In-control \\
\hline 11 & 15 & 1115 & $\begin{array}{ll}0.1 \\
\end{array}$ & 10 & $\begin{array}{l}3 \\
\end{array}$ & -1.6425 & $\begin{array}{ll}0.0807 \\
\end{array}$ & 1.6425 & $\begin{array}{l}1.0472 \\
\end{array}$ & 2.9673 & In-control \\
\hline 12 & 15 & "130 & $\begin{array}{ll}0.1 \\
\end{array}$ & $\begin{array}{l}10.1 \\
\end{array}$ & $\begin{array}{l}3 \\
\end{array}$ & 2.0549 & $\begin{array}{l}-0.0206 \\
\end{array}$ & 2.0549 & $\begin{array}{l}1.0472 \\
\end{array}$ & 2.9673 & In-control \\
\hline 13 & 15 & 145 & 0.1 & 10.2 & $\begin{array}{ll}3 \\
\end{array}$ & 1.5673 & 2.3796 & 2.3796 & $\begin{array}{l}1.0472 \\
\end{array}$ & 2.9673 & $\begin{array}{l}\text { In-control } \\
\end{array}$ \\
\hline 14 & 15 & (160 & 0.1 & 10.3 & $\begin{array}{l}3 \\
\end{array}$ & 2.2500 & "-1.6017 & $\begin{array}{l}2.2500 \\
\end{array}$ & 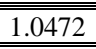 & 2.9673 & In-control \\
\hline 15 & 15 & 175 & 0.1 & 10.4 & $\begin{array}{l}3 \\
\end{array}$ & (1.2529 & -2.5033 & 2.5033 & 1.0472 & 2.9673 & In-control \\
\hline 16 & 15 & 190 & 0.1 & 10.5 & 3 & -2.1290 & $\overline{-1.3716}$ & 2.1290 & 1.0472 & 2.9673 & $\begin{array}{l}\text { In-control } \\
\end{array}$ \\
\hline 17 & 15 & 205 & 0.1 & 10.6 & $\begin{array}{l}3 \\
\end{array}$ & $\begin{array}{l}0.0905 \\
\end{array}$ & 2.9908 & 2.9908 & 1.0472 & 2.9673 & $\begin{array}{l}\text { Out-of-control } \\
\end{array}$ \\
\hline 18 & 15 & 220 & 0.1 & (10.7 & $\begin{array}{l}3 \\
\end{array}$ & (-1.2114 & "-1.1186 & 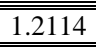 & 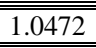 & 2.9673 & I"In-control \\
\hline 19 & 15 & 235 & $\begin{array}{ll}0.1 \\
\end{array}$ & "10.8 & $\begin{array}{l}3 \\
\end{array}$ & $\begin{array}{ll}1.5181 \\
\end{array}$ & - & "1.5181 & $\begin{array}{l}1.0472 \\
\end{array}$ & 2.9673 & I"In-control \\
\hline 20 & 15 & 250 & $\begin{array}{ll}0.1 \\
\end{array}$ & 10.9 & $\begin{array}{l}3 \\
\end{array}$ & "3.5460 & 1.8565 & 3.5460 & $\begin{array}{l}1.0472 \\
\end{array}$ & 2.9673 & Out-of-control \\
\hline
\end{tabular}



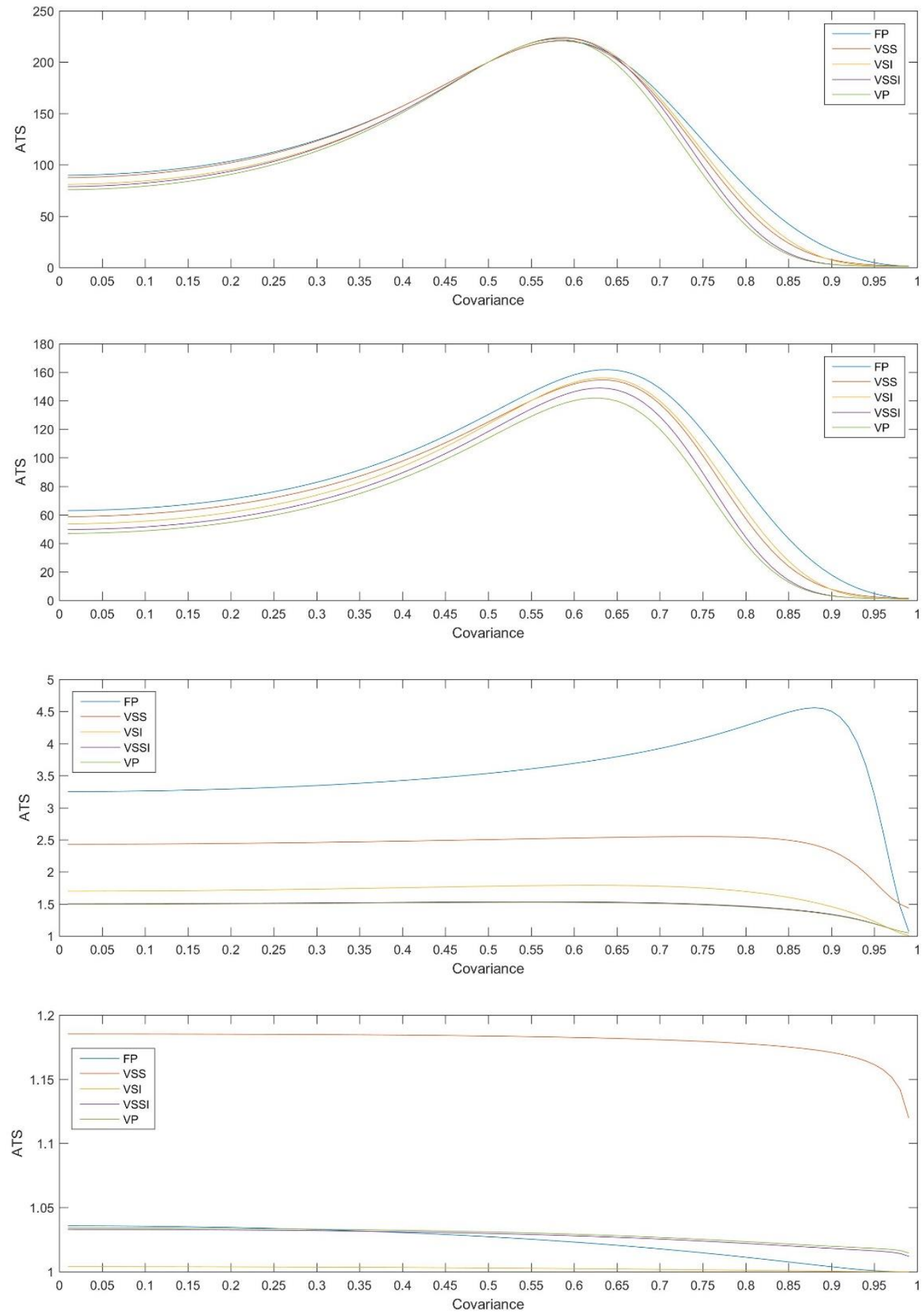

Figure1-ATS vs Covariance $(\theta)$ for zero, small (0.2), medium (0.8) and large (1.5) mean shifts, respectively, for $p=2$ using first scenario case 2 . 

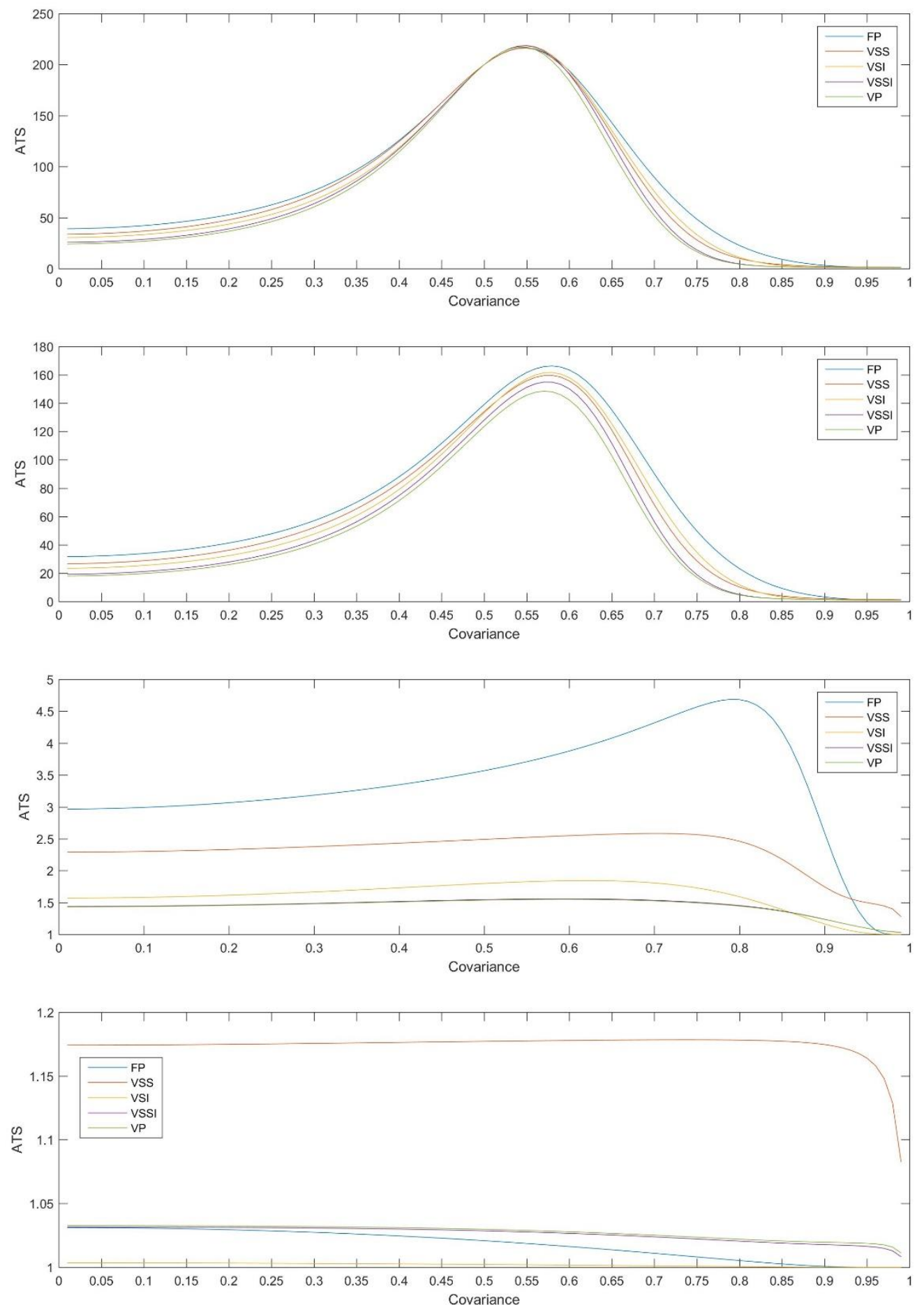

Figure2-ATS vs Covariance ( $\theta$ ) for zero, small (0.2), medium (0.8) and large (1.5) mean shifts, respectively, for $p=3$ using first scenario case 2 . 

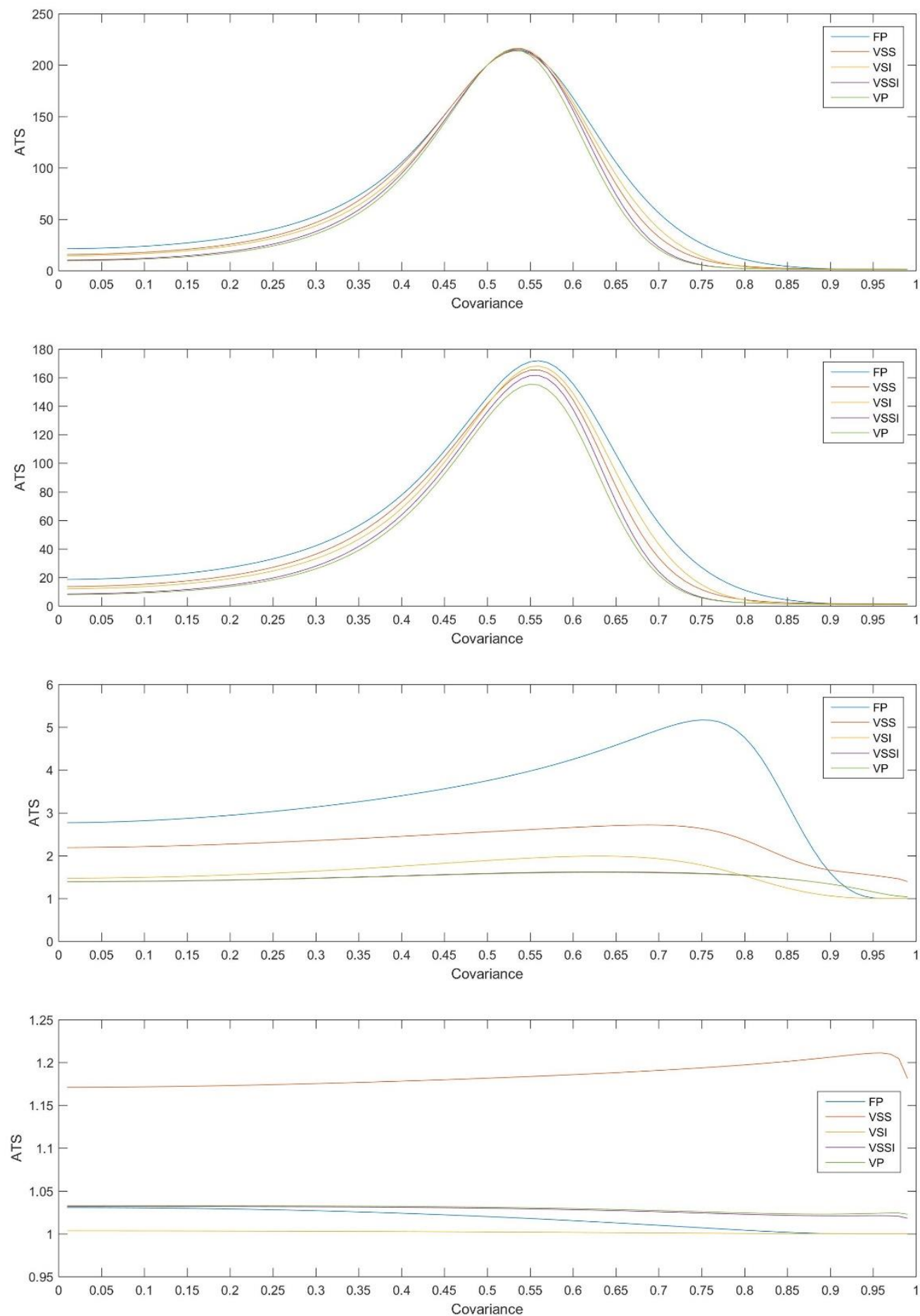

Figure3-ATS vs Covariance $(\theta)$ for zero, small (0.2), medium (0.8) and large (1.5) mean shifts, respectively, for $p=4$ using first scenario case 2 . 


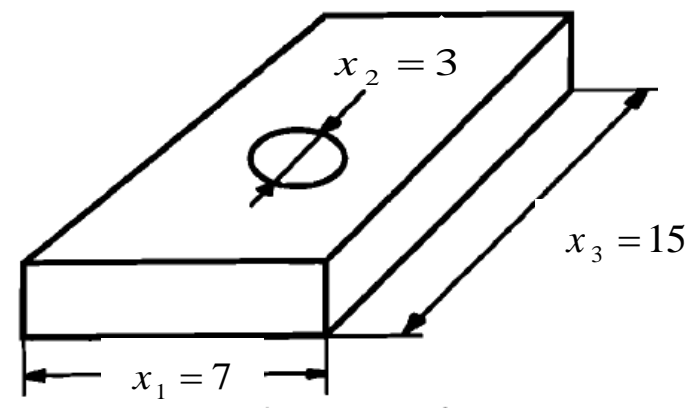

Figure 4- A mechanical part for an illustrative example

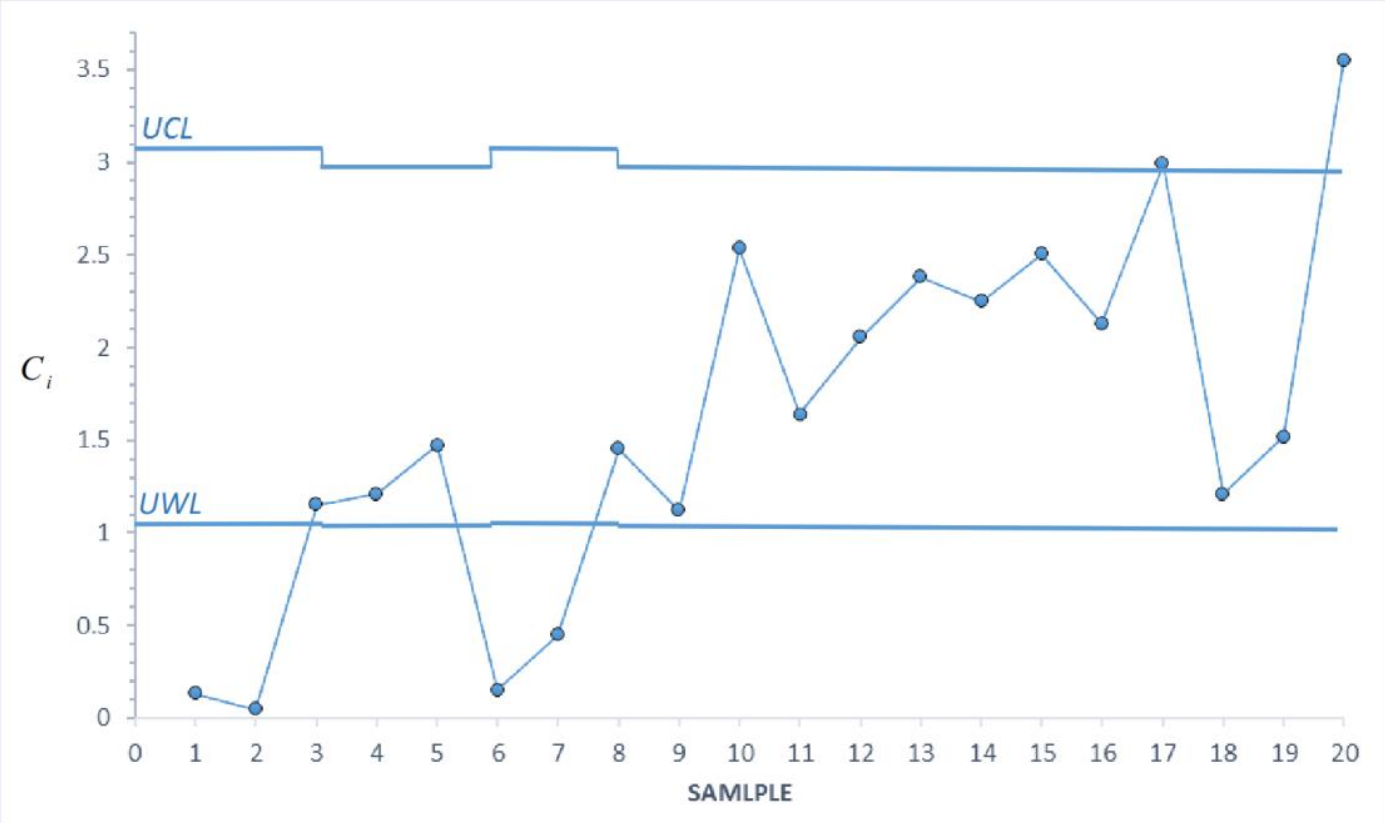

Figure 5- The monitoring VP chart for the illustrative example 